\title{
Acetylcholinesterase Inhibition, Molecular Docking and ADME Prediction Studies of New Dihydrofuran- Piperazine Hybrid Compounds
}

\section{Sait SARI}

Kocaeli University Faculty of science and art, Chemistry Department

Mehmet YILMAZ ( $\square$ mehmet.yilmaz@kocaeli.edu.tr)

Kocaeli University, Faculty of science and arts, Chemistry Department https://orcid.org/0000-00017179-4045

\section{Research Article}

Keywords: Piperazine, Dihydrofuran, Radical cyclization, Acetylcholinesterase inhibition, Molecular docking, ADME

Posted Date: June 10th, 2021

DOI: https://doi.org/10.21203/rs.3.rs-588104/v1

License: (a) (i) This work is licensed under a Creative Commons Attribution 4.0 International License. Read Full License 
Acetylcholinesterase inhibition, molecular docking and ADME prediction studies of new dihydrofuran-piperazine hybrid compounds

Sait SARI ${ }^{1}$ and Mehmet YILMAZ $^{1^{*}}$

$\triangle$ Mehmet YILMAZ

mehmet.yilmaz@kocaeli.edu.tr

Sait SARI

sait.sari@kocaeli.edu.tr

${ }^{1}$ Department of Chemistry, Faculty of Arts and Sciences, Kocaeli University, 41380

Umuttepe, Kocaeli, Turkey 


\begin{abstract}
Novel acrylamide and methacryloyl carrying piperazine-dihydrofuran derivatives (3a-p) were designed and obtained from radical cyclizations of unsaturated piperazine derivatives (1a-f) with 1,3-dicarbonyl compounds (2a-c) mediated by $\mathrm{Mn}(\mathrm{OAc})_{3}$. Structures of obtained compounds were confirmed with ${ }^{1} \mathrm{H}$ NMR (proton nuclear magnetic resonance), ${ }^{13} \mathrm{C}$ NMR (Carbon-13 nuclear magnetic resonance), HRMS (High resolution mass spectrometry), FTIR (Fourier-transform infrared spectroscopy and melting point analysis. Inhibitory activites of all piperazine-dihydrofuran compounds were evaluated against AChE (Acetylcholinesterase) by Ellman method and test results showed that $\mathbf{3 a}, \mathbf{3 c}, \mathbf{3 j}$ and $\mathbf{3 I}$ are most active AChEI's (AChE inhibitors) of our work with $\mathrm{IC}_{50}$ (half-maximal inhibitory concentration) values of 2.62, 5.29, 1.17 and $3.90 \mu \mathrm{M}$, respectively. Furthermore, ligand-protein interactions and inhibitory activity mechanisms of $\mathbf{3 a}$ and $\mathbf{3 j}$ were investigated by molecular docking. Finally, in silico molecular property and ADME (absorption, distribution, metabolism and excretion) of potential AChEI's (AChE inhibitor) were predicted by PreADMET and Molinspiration webservers. It can be concluded that the lead compound $\mathbf{3 j}$ show excellent inhibiton and satisfactory druglike characteristics.
\end{abstract}

\title{
Keywords
}

Piperazine, Dihydrofuran, Radical cyclization, Acetylcholinesterase inhibition, Molecular docking, ADME 


\section{Introduction}

Alzheimer's disease (AD) is a neurodegenerative disorder and is one of the main cause of dementia effecting elderly people. The illness is characterized with executive disorders, memory loss, mood disturbances, depression and progressive loss of cognitive abilities [1,2]. Based on the report of World Health Organization (WHO) about AD, about 36 million people around globe were suffering from dementia until 2010 and this would be increased to 66 million by 2030 [3]. Many theories were suggested to clarify the exact origin of AD such as cholinergic transmission [4], tau protein hyperphosphorylation [5] and beta-amyloid aggregation [6]. Among them cholinergic transmission is the most commonly accepted theory and increasing levels of neurotransmitter acetylcholine in brain is crucial for the treatment of AD [7-9].

Acetylcholinesterase (AChE) is an enzyme in cholinesterase family which catalyzes the rapid hydrolysis of neurotransmitter acetylcholine and terminates impulse transmission at cholinergic synapses [10]. Inhibiting AChE is the most prominent way in the field of AD treatment [11] and there are many commercially available inhibitor drugs such donepezil [12], rivastigmine [13] and galantamine [14].

Heterocycles bearing nitrogen are important compounds in the field of medicinal chemistry and widely used for their biological properties [15]. Piperazine is considered a privileged structure for its ability of binding to multiple structures with high affinity [16]. There are many biological activities reported for compounds bearing piperazine moieties such as anticonvulsant [17], antibacterial [18], antituberculosis [19], antiviral [20], anticancer [21] and antimalarial [22]. Also many AChE inhibition studies were performed for piperazine derivatives [23-25].

Dihydrofurans are biologically active heterocycles and useful building blocks for naturally occurring compounds such as Sarcophytoxide [26], Clerodin [27], Fercoprolone [28] and Austocystin [29]. Dihydrofurans can be obtained by C-C bond forming radical cyclization reactions that occur through the addition of $\alpha$-carbon radicals to unsaturated systems [30-32]. 
Alpha carbon radicals are generated from active methylene compounds via single electron transferring transition metal salts $\left(\mathrm{Mn}^{3+}, \mathrm{Ce}^{4+}, \mathrm{Co}^{3+}\right.$, etc). Manganese(III) acetate [33-37] and Cerium(IV) ammonium nitrate [38-40] are the most widely used transition metal salts in this reaction. Our research group has reported the synthesis of dihydrofuran compounds by the radical cyclization of 1,3-dicarbonyl compounds with various unsaturated systems such as aromatic conjugated alkenes $[41,42]$, dienes $[43,44]$ and conjugated amide derivatives $[45,46]$ mediated by Manganese(III) acetate and Cerium(IV) ammonium nitrate.

Over recent years there are some works in literature about AChE inhibition of acrylamide and acrylamide containing piperazine compounds. Pan and coworkers described the synthesis of ferulic acid-memoquin hybrids which contains aromatic acrylamide moeities and evaluated their inhibiton capabilities against $\mathrm{AChE}$ and reported $\mathrm{IC}_{50}$ values between 3.2-34.7 $\mu \mathrm{M}$ [47]. Additionally, Shaik and coworkers designed flavone-8-acrylamide compounds and obtained inhibition results between 0.064-2.81 $\mu \mathrm{M}$ [48]. Moreover, cinnamic N-benzylpiperidine hybrids were synthesized by Estrada et al. and they obtained good inhibition results $\left(\mathrm{IC}_{50}=0.26\right.$ 8.73) [49]. Finally aromatic acrylamide carrying piperazine derivatives were obtained by Singh and coworkers that show $\mathrm{AChE}$ inhibition with $\mathrm{IC}_{50}$ values between 9.91-29.34 $\mu \mathrm{M}$ [50].

Our research group reported aromatic amide substituted piperazine-dihydrofuran derivatives [51] and good inhibition results were reported ( $\mathrm{IC}_{50}$ values between 2.24-17.93 $\mu \mathrm{M}$ ). Encouraged by the results of our previous work and based on the results that were acquired by many research groups for acrylamides and piperazine compounds over recent years, in present work we studied the radicalic cyclization reaction between aromatic substituted acrylamide and methacryloyl carrying piperazine compounds (1a-f) with enolizable 1,3-dicarbonyls (dimedone (2a), acetylacetone (2b), ethylacetoacetate (2c)) via $\mathrm{Mn}(\mathrm{OAc})_{3}$. Radicalic cyclization is possible to occur on both methacryloyl and substituted acrylamide sides depending on steric hindrance and radical stability. Mechanism of this reaction is discussed in Results and 
Discussion section. General molecular structures of obtained piperazine-dihydrofuran molecules can be seen in Scheme 1. Acrylamide and substituted acrylamide carrying piperazine-dihydrofuran products were obtained from these reactions and both of these final products (3a-p) were isolated and evaluated for AChE inhibitions.

A

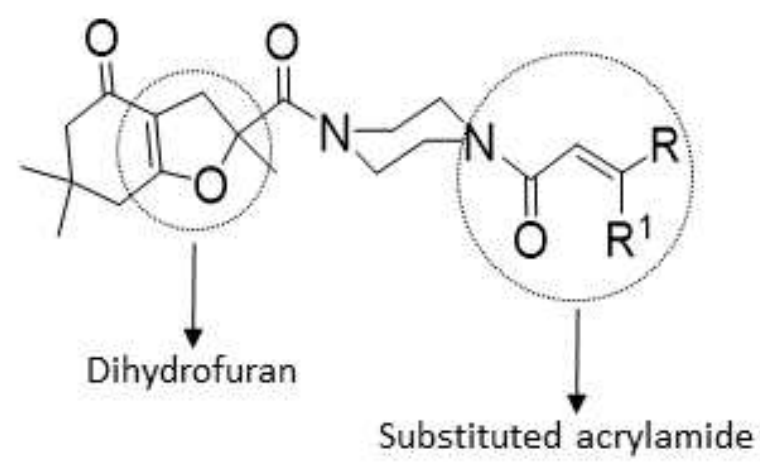

B

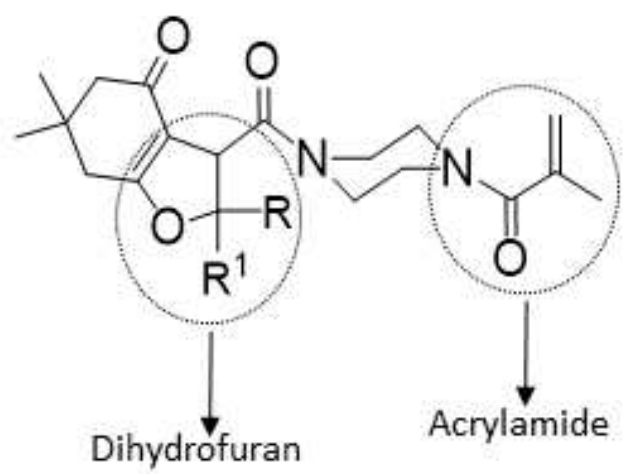

R: Phenyl, Thiophene, Furyl

$\mathrm{R}^{1}$ : Methyl, Phenyl, $\mathrm{H}$

Scheme 1 Molecular structures of substituted acrylamide (A) and methacryoyl (B) carrying piperazine-dihydrofuran compounds

To further understand the interaction of newly synthesized compounds with AChE, molecular docking studies were performed to investigate the binding modes with AChE (PDB: 4EY7) active site.

Moreover, to predict the druglike potentials of obtained compounds, in silico molecular property and ADME (absorption, distribution, metabolism, excretion) prediction studies were conducted to predict druglikeness of obtained compounds by using Molinspiration and PreADMET online servers. 


\section{Results and Discussion}

\section{Chemistry}

The reactions of acrylamide substituted piperazines (1a-e) with dimedone (2a) and acetylacetone (2b) were given at Table 1. The reaction of piperazine derivative 1a with dimedone (2a) gave piperazine substituted dihydrofuran compounds 3a (10\%) and $\mathbf{3 b}(45 \%)$ from the cyclization of each acyl group and these compounds were differantiated by their ${ }^{1} \mathrm{H}$ NMR spectra. The ${ }^{1} \mathrm{H}$ NMR spectrum of 3a shows trans alkene protons at 6.85 and $7.72 \mathrm{ppm}$ as doublet $(J=15.6 \mathrm{~Hz})$ for each proton. Also, geminal protons of dihydrofuran ring can be seen at 2.72 and $3.50 \mathrm{ppm}$ as doublet $(J=15.2 \mathrm{~Hz})$ for each proton. The terminal alkene protons of $\mathbf{3 b}$ is resonated at 5.03 and $5.20 \mathrm{ppm}$ as two singlet. Also, vicinal protons of dihydrofuran moeity of $\mathbf{3 b}$ can be seen at 4.23 and $6.11 \mathrm{ppm}$ as two doublet $(J=5.6 \mathrm{~Hz})$.

Reactions of piperazine derivatives $\mathbf{1 b}$ and $\mathbf{1 c}$ with dimedone (2a) gave acrylamide piperazine substituted dihydrofurans 3c $(45 \%)$ and 3d (60\%), respectively. Both radical cyclizations occurred through the methacryloyl group, regioselectively. However, the reaction of methacryloyl and (2E),(4E)-5-phenyl-2,4-pentadienoyl substituted compound 1d with 2a formed compound 3e (20\%) through 2,4-pentadienoyl. The exact structure of this compound was clarified with ${ }^{1} \mathrm{H}$ NMR and HMBC spectra. Also, the reaction of $1 \mathbf{e}$ with $\mathbf{2 a}$ gave piperazine substituted dihydrofurans $\mathbf{3 f}(10 \%)$ and $\mathbf{3 g}(40 \%)$ from the cyclizations of each acyl group on 1e. In addition, $\mathbf{3 h}(45 \%)$ and $\mathbf{3 i}(30 \%)$ were obtained from the reaction of acetylacetone (2b) with 1a and 1c, respectively. 
Table 1 Synthesis of piperazine-dihydrofurans (3a-i).

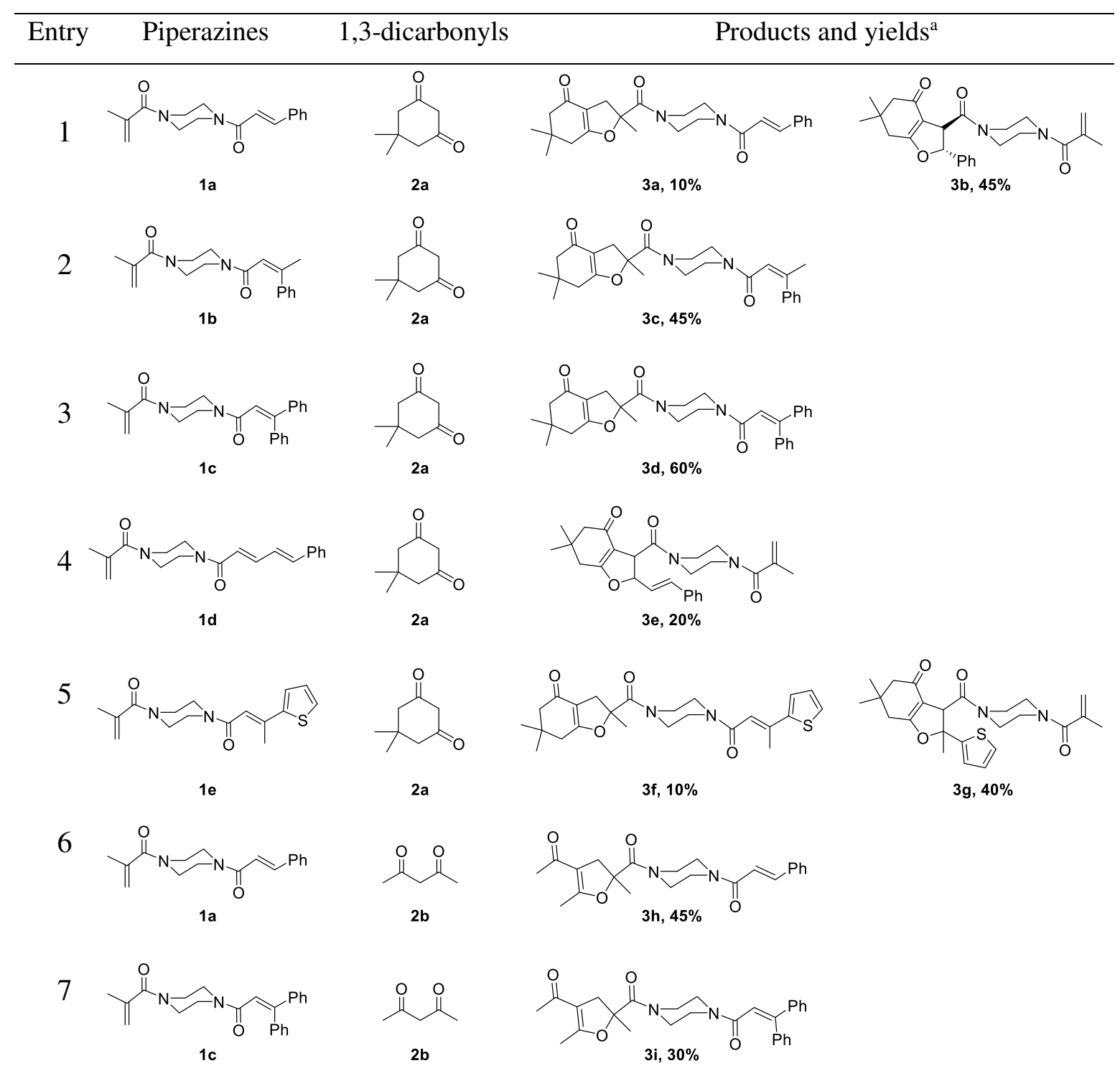

a) Isolated yields based on 1,3-dicarbonyl compounds

As can be seen at Table 2, while piperazine-dihydrofurans $\mathbf{3 j}$ (13\%) and $\mathbf{3 k}$ (25\%) were obtained from the cyclization (through both acyl groups) of $\mathbf{1 a}$ with $\mathbf{2 c}, \mathbf{3 1}$ (40\%) and $\mathbf{3 m}$ (50\%) were isolated from the cyclization (only through methacryloyl group) of $\mathbf{1 b}$ and $\mathbf{1 c}$ with $\mathbf{2 c}$. Similarly, while the reaction of $\mathbf{1 f}$ with $\mathbf{2 c}$ gave piperazine dihydrofurans $\mathbf{3 n}(20 \%)$ and $\mathbf{3 o}$ (30\%), only $\mathbf{3 p}(20 \%)$ was isolated from the reaction of $\mathbf{1 e}$ with $\mathbf{2 c}$. 
Table 2 Synthesis of piperazine-dihydrofurans (3j-p).

Entry

a) Isolated yields based on 1,3-dicarbonyl compounds.

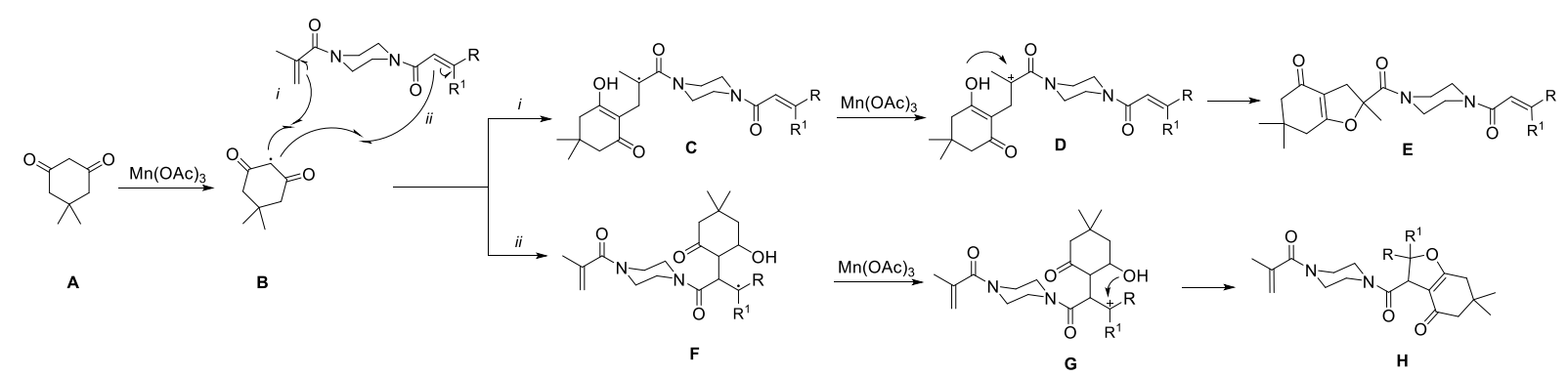

Scheme 2 Proposed mechanism of $\mathrm{Mn}(\mathrm{OAc})_{3}$ mediated radical cyclization.

The proposed mechanism for the formation of dihydrofurans is explained in Scheme 2 [52]. According to this mechanism, the enol form of dimedone (A) reacts with $\mathrm{Mn}(\mathrm{OAc})_{3}$ and an alpha carbon radical $\mathbf{B}$ is formed, while $\mathrm{Mn}^{3+}$ reduces to $\mathrm{Mn}^{2+}$. Alpha carbon radical can interact with both unsaturated sides of piperazine compound and both of these pathways ( $i$ and $i i)$ are likely to occur at the same time. On pathway $i$ an electron from alkene is added to this $\alpha$-carbon 
radical and produces the radical carbon intermediate $\mathbf{C}$. Intermediate $\mathbf{C}$ oxidizes to carbocation D with $\mathrm{Mn}(\mathrm{OAc})_{3}$ and intramolecular cyclization of $\mathbf{D}$ forms the product $\mathbf{E}$. On pathway $i i$ radicalic cyclization reaction follows similar steps on the other unsaturated site and product $\mathbf{H}$ is formed.

\section{In vitro inhibition results of piperazine-dihydrofuran compounds against $\mathrm{AChE}$}

Starting acylated piperazine derivatives (1a-f) used in this study were tested against AChE and they show almost no inhibition $\left(\mathrm{IC}_{50}>100 \mu \mathrm{M}\right)$. On the other hand, in vitro inhibition capabilities of some of the obtained piperazine substituted dihydrofurans were proved to be significantly high. All results compared to standard drugs Donepezil and Galanthamine were presented in Table 3.

Table 3 IC $_{50}$ values of piperazine-dihydrofuran compounds (3a-p) towards AChE.

\begin{tabular}{cc}
\hline Compound & IC50 \pm SD $(\mu \mathrm{M})^{\mathrm{a}}$ \\
\hline $\mathbf{3 a}$ & $2.62 \pm 0.2$ \\
$\mathbf{3 b}$ & $>100$ \\
$\mathbf{3 c}$ & $5.29 \pm 0.5$ \\
$\mathbf{3 d}$ & $11.89 \pm 1.3$ \\
$\mathbf{3 e}$ & $>100$ \\
$\mathbf{3 f}$ & $8.55 \pm 0.6$ \\
$\mathbf{3 g}$ & $>100$ \\
$\mathbf{3 h}$ & $>100$ \\
$\mathbf{3 i}$ & $>100$ \\
$\mathbf{3 j}$ & $1.17 \pm 0.7$ \\
$\mathbf{3 k}$ & $>100$ \\
$\mathbf{3}$ & $3.90 \pm 0.5$ \\
$\mathbf{3 m}$ & $8.36 \pm 0.4$ \\
$\mathbf{3 n}$ & $6.11 \pm 0.6$ \\
$\mathbf{3 0}$ & $>100$ \\
$\mathbf{3 p}$ & $8.42 \pm 0.3$ \\
Donepezil[53] & 0.041 \\
Galanthamine[53] & 1.65 \\
\hline
\end{tabular}

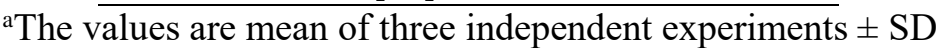

$\mathrm{IC}_{50}$ values of cinnamoyl acrylamide substituted $\mathbf{3 a}$ and methacryloyl substituted $\mathbf{3 b}$ were calculated and while $\mathbf{3 a}$ has $\mathrm{IC}_{50}$ value of $2.62 \mu \mathrm{M}, \mathbf{3} \mathbf{b}$ has almost no inhibition $\left(\mathrm{IC}_{50}>100\right.$ $\mu \mathrm{M})$. Also, $\mathrm{IC}_{50}$ values for compounds $\mathbf{3 c}$ and $\mathbf{3 d}$ are 5.19 and $11.89 \mu \mathrm{M}$, respectively. By comparing these $\mathrm{IC}_{50}$ values with $\mathbf{3 a}\left(\mathrm{IC}_{50}=2.62 \mu \mathrm{M}\right)$ it can be seen that inhibition powers of 
3c and $\mathbf{3 d}$ are lower than $\mathbf{3 a}$. In addition, while $\mathrm{IC}_{50}$ value of $\mathbf{3 f}$ was calculated as $8.55 \mu \mathrm{M}$, it is $>100 \mu \mathrm{M}$ for methacryloyl containing compound 3g. Similarly, compound 3e shows almost no inhibition $\left(\mathrm{IC}_{50}>100 \mu \mathrm{M}\right)$. Also, contrary to products obtained from dimedone, $\mathbf{3 h}$ and $\mathbf{3 i}$, which obtained from the reactions of acetylacetone $(\mathbf{2 b})$, show no inhibition effects.

Among the piperazine-dihydrofuran compounds obtained from the reactions of ethyl acetoacetate (2c) with acrylamide piperazines (1a-e), compounds $\mathbf{3 j}\left(\mathrm{IC}_{50}=1.17 \mu \mathrm{M}\right)$ and $\mathbf{3 l}$ $\left(\mathrm{IC}_{50}=3.90 \mu \mathrm{M}\right)$ show best inhibition effects. Also, IC $\mathrm{IC}_{50}$ values of $\mathbf{3 m}, \mathbf{3 n}$ and $\mathbf{3 p}$ were calculated as $8.36,6.11$ and $8.42 \mu \mathrm{M}$, respectively. On the other hand, similar to compounds mentioned above, methacryloyl containing compounds $\mathbf{3 k}$ and $\mathbf{3 o}$ has almost no inhibition effect $\left(\mathrm{IC}_{50}>100 \mu \mathrm{M}\right)$. In the light of these informations, it is clear that, aromatic moeity carrying acrylamide substituents on piperazine-dihydrofuran compounds have significantly positive effect on inhibitions. Also, it is concluded that, carboxylate substitution on dihydrofuran group increases inhibition efficiency than other substitutions on dihydrofurans. Based on these results, compound $\mathbf{3} \mathbf{j}$ is selected as our lead compound.

\section{Molecular docking results of selected piperazine-dihydrofuran compounds (3a and 3j)}

Molecular docking studies were performed on two of our most potent inhibitor compounds (3a and 3j). Docking procedure was validated by re-docking the native ligand donepezil to target AChE. Near perfect alignment with a RMSD value of 0.340 was obtained from validation results. Binding score of native ligand donepezil is $-12.2 \mathrm{Kcal} / \mathrm{mol}$. Binding energies for top docking poses of ligands 3a and $\mathbf{3 j}$ are -9.6 and $-10.4 \mathrm{Kcal} / \mathrm{mol}$, respectively. Docking poses of 3a, 3j and Donepezil are superpositioned in AChE active site cavity and shows good alignment with native ligand Donepezil. 


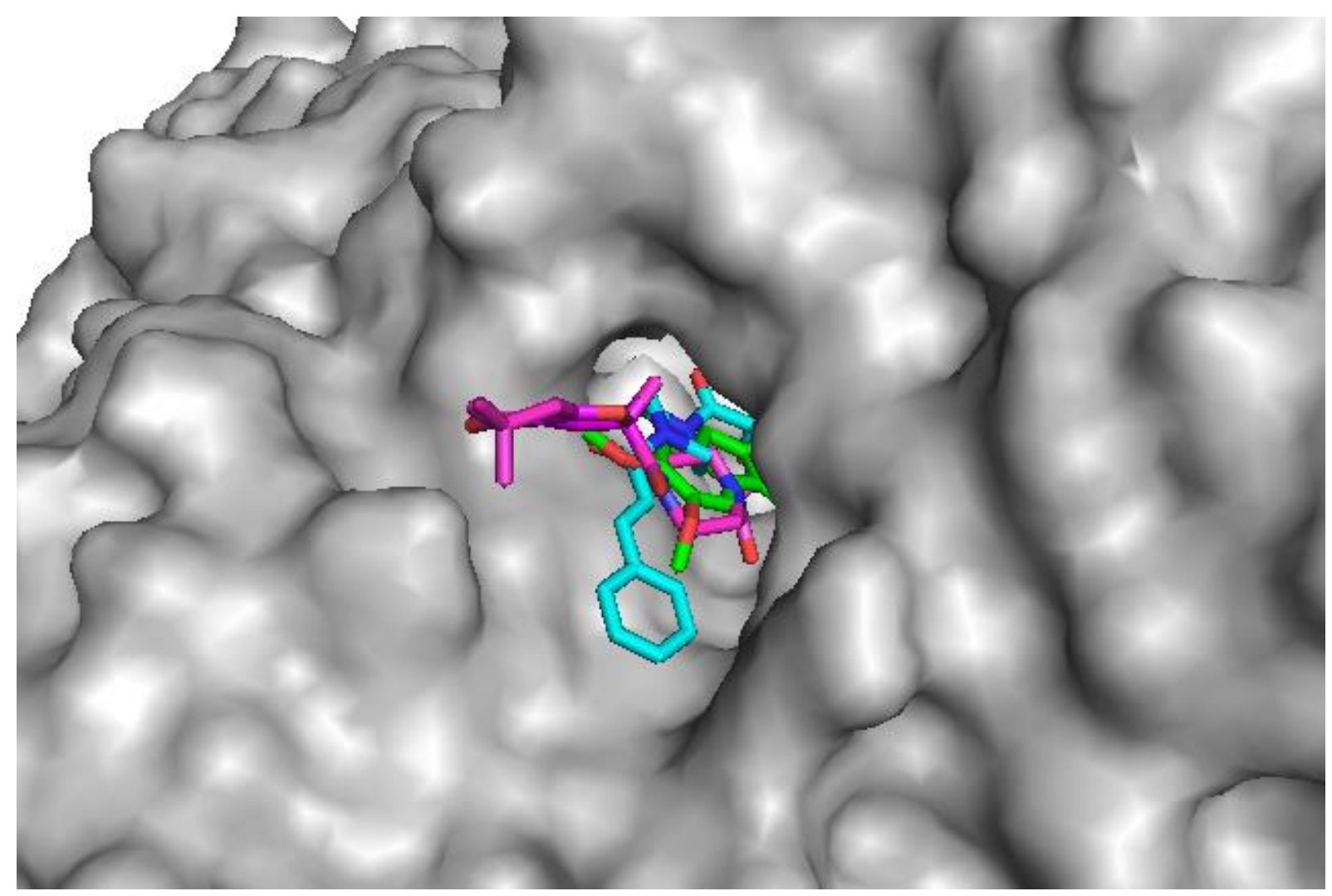

Fig. 1 AChE active site cavity with donepezil (green), $\mathbf{3 a}$ (magenta) and $\mathbf{3 j}$ (cyan) inside.

Ligand-protein interactions of top binding poses of ligands $\mathbf{3 a}$ and $\mathbf{3 j}$ were given at Figure 2 . By investigating the docking mod of $\mathbf{3 a}$ it can be seen that one of the methyls of dimedone ring of 3a forms $\pi$-alkyl interactions with aromatic moeity of HIS287. Also, dimedone carbonyl forms a carbon-hydrogen bond with TRP286. Methyl group on dihydrofuran ring interacts with TYR341 through a hydrophobic $\pi$-alky interaction. Piperazine ring forms hydrophobic $\pi$-alkyl interactions with aromatic moeities of TRP286 and carbon-hydrogen bonds with SER293 and TYR341. Additionally acrylamide carbonyl forms hydrogen bondings with PHE295 and ARG296. Finally aromatic moeity of acrylamide group forms $\pi-\pi$ interactions with TYR341 and PHE338.

By investigating the interactions of $\mathbf{3} \mathbf{j}$, it can be seen that ethyl carboxylate moeity of $\mathbf{3} \mathbf{j}$ forms $\pi-\sigma$ interaction with aromatic moeity of TRP86. Also ester carbonyl forms a Carbon-Hydrogen bond with HIS447. Methyl groups on dihydrofuran ring made $\pi$-alkyl interactions with 
PHE338, TYR124 and PHE297, also one methyl group on dihydrofuran ring interacts with TYR341 through a $\pi-\sigma$ interaction. Moreover carbonyl next to dihydrofuran ring forms a hydrogen bond with TYR124. Additionally, piperazine ring interacts with TYR341 and TRP286 through $\pi$-alkyl interactions. Finally, aromatic ring of cinnamoyl group interacts with LEU289 through a $\pi$-alkyl interaction.

By considering the ligand-protein interactions of top binding modes of ligands it can be seen that docking results support in vitro inhibition results.
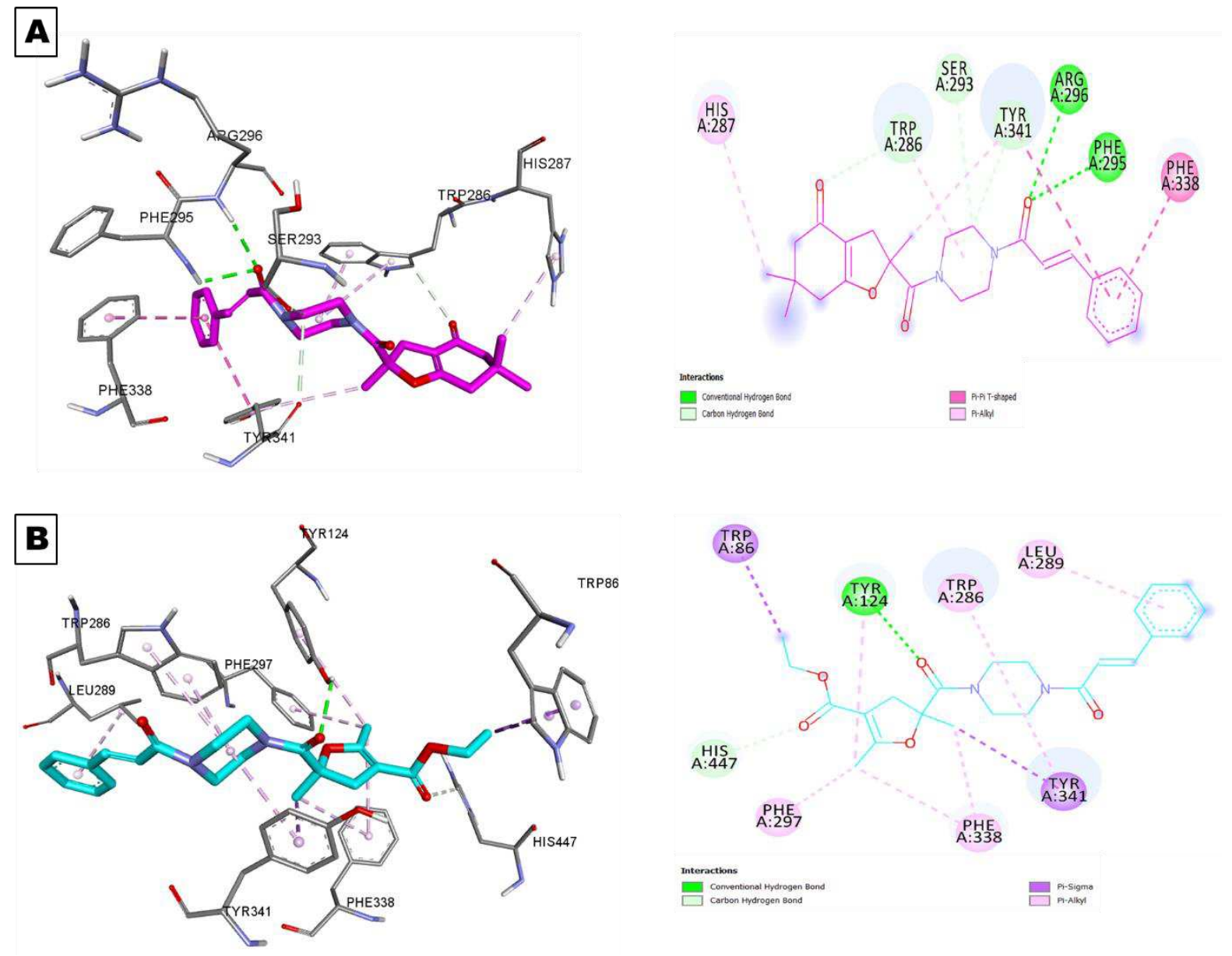

Fig. 2 Ligand-protein interactions of $\mathbf{3 a}(\mathbf{A})$ and $\mathbf{3 j}$ (B).

\section{In silico molecular property and ADME prediction results}

ADME properties are one of the main reasons for a drug candidate to fail in clinical trials. In silico molecular property and ADME predictions of obtained piperazine-dihydrofuran compounds which have inhibition powers against AChE (AChEI's) (3a, 3c, 3d, 3f, 3j, 31, 3m, 
3m and 3p) were carried out using Molinspiration (https://www.molinspiration.com/) and PreADMET (https://preadmet.bmdrc.kr/) webservers in order to predict druglikeness of these molecules. According to Lipinski's rule [54] a drug candidate has no more than one violation of the following criteria: (i) Hydrogen bond acceptors must be $\leq 10$. (ii) Hydrogen bond donors must be $\leq 5$ (iii) Molecular weight (MW) must be less than $500 \mathrm{D}$ and (iv) Octanol-water partition coefficient (MilogP) of the molecule must be $\leq 5$.

As can be seen in Table 4 Piperazine-dihydrofuran compounds show no violation against Lipinski’s rule.

Table 4 In silico molecular property predictions for AChEI's.

\begin{tabular}{llllllll}
\hline Code & MW $^{\mathrm{a}}$ & MiLogP $^{\mathrm{b}}$ & $\mathrm{HBA}^{\mathrm{c}}$ & $\mathrm{HBD}^{\mathrm{d}}$ & Nviol $^{\mathrm{e}}$ & $\mathrm{TPSA}^{\mathrm{f}}$ & $\mathrm{MVol}^{\mathrm{g}}$ \\
\hline Rule & $<500$ & $\leq 5$ & $\leq 10$ & $\leq 5$ & $\leq 1$ & $<160 \AA^{2}$ & \\
3a & 422.52 & 3.26 & 6 & 0 & 0 & 66.92 & 398.16 \\
3c & 436.55 & 3.14 & 6 & 0 & 0 & 66.92 & 414.72 \\
3d & 498.62 & 4.36 & 6 & 0 & 0 & 66.92 & 469.57 \\
3f & 442.58 & 3.04 & 6 & 0 & 0 & 66.92 & 405.43 \\
3j & 412.49 & 3.15 & 7 & 0 & 0 & 76.16 & 384.68 \\
31 & 426.51 & 3.03 & 7 & 0 & 0 & 76.16 & 401.24 \\
3m & 488.58 & 4.25 & 7 & 0 & 0 & 76.16 & 456.09 \\
3n & 418.51 & 2.87 & 7 & 0 & 0 & 76.16 & 375.39 \\
3p & 432.54 & 2.93 & 7 & 0 & 0 & 76.16 & 391.95 \\
\hline
\end{tabular}

a: Molecular weight (MW); b: logarithm of octanol-water partition coefficient (MiLogP); c: Number of hydrogen bond acceptors (HBA); d: Number of hydrogen bond donors (HBD); e: Lipinski rule violations (nviol); f: Topological polar surface area (TPSA); g: Molecular volume (MVol). 
Moreover, in silico ADME prediction results of AChEI's were given at Table 5. [55]

Table 5 In silico ADME predictions of AChEI's.

\begin{tabular}{|c|c|c|c|c|c|}
\hline & \multicolumn{4}{|l|}{ Absorption } & \multirow{2}{*}{$\begin{array}{l}\text { Distribution } \\
\text { In vitro plasma } \\
\text { protein binding } \\
\text { PPB }(\%)\end{array}$} \\
\hline Code & $\begin{array}{l}\text { Human } \\
\text { intestinal } \\
\text { absorption (\%) }\end{array}$ & $\begin{array}{l}\text { In vitro Caco-2 } \\
\text { cell } \\
\text { permeability } \\
(\mathrm{nm} / \mathrm{s})\end{array}$ & $\begin{array}{l}\text { In vitro MDCK cell } \\
\text { permeability }(\mathrm{nm} / \mathrm{s})\end{array}$ & $\begin{array}{l}\text { In vitro skin } \\
\text { permeability } \\
(\log \mathrm{Kp}, \mathrm{cm} / \mathrm{h})\end{array}$ & \\
\hline Rule & $\begin{array}{l}0-20 \text { (poor) } \\
20-70 \\
\text { (moderate) } \\
70-100 \text { (well) }\end{array}$ & $\begin{array}{l}<4 \text { (low), } 4-70 \\
\text { (moderate), }>70 \\
\text { (high) }\end{array}$ & $\begin{array}{l}<25 \text { (low) 25-500 } \\
\text { (moderate) }>500 \\
\text { (high) }\end{array}$ & & $\begin{array}{l}>90 \text { (strongly } \\
\text { bound) }<90 \\
\text { (weakly bound) }\end{array}$ \\
\hline $\mathbf{3 a}$ & 98.00 & 49.67 & 0.06 & -3.29 & 82.79 \\
\hline $3 c$ & 97.86 & 50.32 & 0.06 & -3.25 & 84.87 \\
\hline 3d & 97.48 & 52.53 & 0.04 & -2.39 & 90.62 \\
\hline $3 f$ & 99.52 & 53.17 & 0.07 & -3.85 & 84.09 \\
\hline 3j & 98.95 & 45.52 & 0.11 & -3.17 & 76.39 \\
\hline 31 & 98.87 & 46.87 & 0.08 & -3.14 & 79.85 \\
\hline $3 m$ & 97.69 & 50.02 & 0.04 & -2.36 & 89.16 \\
\hline $3 n$ & 98.65 & 50.25 & 0.17 & -3.75 & 72.94 \\
\hline $3 p$ & 98.96 & 51.48 & 0.11 & -3.71 & 78.50 \\
\hline
\end{tabular}

Human intestinal absorption (HIA) indicates gastrointestinal permeation across membranes for drugs which taken orally. All AChEI compounds show great HIA values over $97 \%$.

In vitro Caco-2 cell permeability is an indication of intestinal absorption of drugs. According to our results all AChEI's show moderate permeations between $41-53 \mathrm{~nm} / \mathrm{s}$.

In vitro MDCK cell permeability test utilizes canine kidney cells to test permeability. All AChEI test compounds show low permeation values.

Skin permeability is a factor that indicates delivery of a drug through transdermal administration. All AChEI's compounds show negative permeability which shows transdermal administration is not suitable for these molecules.

In vitro plasma protein binding (PPB) indicates percentage of a drug is bound to blood plasma proteins. Our AChEI's show binding values less than $90 \%$ which means they can efficiently diffuse to cell membranes. 


\section{Conclusion}

In the presented work, new piperazine-dihydrofuran compounds (3a-p) were designed and synthesized from $\mathrm{Mn}(\mathrm{OAc})_{3}$ mediated radical cyclizations of 1,3-dicarbonyl compounds (2a-c) and acrylamide carrying piperazine derivatives (1a-f) in low and medium yields. AChE inhibition capabilities of starting piperazine derivatives (1a-f) and piperazine-dihydrofuran compounds (3a-p) were tested. Although many of the piperazine-dihydrofuran compounds (3a, 3c, 3d, 3f, 3j, 3l, 3m, 3n and 3p) show inhibition capabilities against AChE, starting acylated piperazine compounds (1a-f) show no inhibition effects. While piperazine-dihydrofuran compounds containing aromatically substituted acrylamide moeities has high inhibition effects against $\mathrm{AChE}$ ( $\mathrm{IC}_{50}$ values ranging from 1.17 to $11.89 \mu \mathrm{M}$ ) methacryloyl carrying piperazinedihydrofuran compounds (3b, 3g, 3h, 3i, 3k and 3o) show almost no inhibitions. Also, carbethoxy substituted piperazine-dihydrofuran compounds show higher inhibition effects than other piperazine-dihydrofurans, especially $\mathbf{3} \mathbf{j}\left(\mathrm{IC}_{50}=1.17 \mu \mathrm{M}\right)$ which is our lead compound. In addition, molecular docking studies were performed with the lead compound $\mathbf{3 j}$ and the other most potent AChEI 3a to investigate ligand-protein interactions and binding energies. Calculated docking results were compared to standart drug Donepezil. Binding scores of Donepezil is $-12.2 \mathrm{Kcal} / \mathrm{mol}$ and $-9.6,-10.4 \mathrm{Kcal} / \mathrm{mol}$ for $\mathbf{3 a}$ and $\mathbf{3 j}$ respectively. Finally, in silico molecular property analysis and ADME prediction studies show that our lead compound 3j and other AChEI's have satisfactory druglike characteristics. Summarily, the lead piperazine-dihydrofuran compound $\mathbf{3 j}$ which carries phenyl substituted acrylamide moeity and carboxylate group have excellent AChE inhibition and satisfactory druglike characteristics. This compound have potential to be a drug candidate and can be further modified to increase the activity against Acetylcholinesterase. 


\section{Experimental}

All reagents and solvents are commercially available and analitically pure unless otherwise stated. AChE (from electric eel, type V-S), acetylthiocholine iodide (ATCI), 5,5'-dithiobis(2nitrobenzoic acid) (DTNB) were supplied from Sigma Aldrich. Radical oxidant $\mathrm{Mn}(\mathrm{OAc})_{3}$ was synthesized by electrochemical method [43].

${ }^{1} \mathrm{H}$ NMR and ${ }^{13} \mathrm{C}$ NMR spectra were recorded on a Varian Mercury-400 High performance Digital FT-NMR and Varian Oxford NMR300 spectrometers. HRMS spectra were obtained on an Agilent 1200/6210 LC/MS spectrophotometer. IR spectra (ATR) were obtained with a Bruker Tensor27 spectrophotometer in the $400-4000 \mathrm{~cm}^{-1}$ range with $2 \mathrm{~cm}^{-1}$ resolutions. UV absorbance was recorded by Rigol Ultra-3000 UV-Vis Spectrophotometer. Melting points were determined on a Gallenkamp capillary melting point apparatus. Thin layer chromatography (TLC) was performed on Merck aluminum-packed silica gel plates. Purification of products were performed by column chromatography on silica gel (Merck silica gel 60, 40-60 $\mu \mathrm{m}$ ) or preparative TLC on silica gel of Merck (PF254-366 nm).

\section{General synthesis procedure and spectroscopic data of piperazine dihydrofuran compounds (3a-p)}

Starting unsaturated piperazine derivatives (1a-f) were obtained according to our previously reported work [56]. All piperazine-dihdydrofuran compounds (3a-p) were synthesized by the general method described below.

$\left[\mathrm{Mn}(\mathrm{OAc})_{3}\right] \cdot 2 \mathrm{H}_{2} \mathrm{O}(2 \mathrm{mmol}, 0.53 \mathrm{~g})$ in $15 \mathrm{~mL}$ glacial acetic was heated to $80^{\circ} \mathrm{C}$ until dissolved. After that the solution temperature was cooled to $65{ }^{\circ} \mathrm{C}$ and a solution of 1,3-dicarbonyl compound (2a-c) (1mmol) and piperazine compound (1a-f) $(1.2 \mathrm{mmol})$ in $3 \mathrm{~mL}$ of acetic acid was added. The mixture was stirred and disappearance of the initial dark brown indicated that the reaction was finished (10-30 $\mathrm{min})$. After that, water was added and the reaction mixture was extracted with $\mathrm{CHCl}_{3}(3 \times 20 \mathrm{~mL})$. The combined organic phase was neutralised with saturated 
$\mathrm{NaHCO}_{3}$ solution, dried over anhydrous $\mathrm{Na}_{2} \mathrm{SO}_{4}$ and evaporated. The crude product was purified with column chromatography or preperative TLC (chloroforom:acetone $(85: 15)$ as eluent).

\section{2-(4-cinnamoylpiperazine-1-carbonyl)-2,6,6-trimethyl-3,5,6,7-tetrahydrobenzofuran-} $4(2 H)$-one (3a)

It was obtained as a yellow oil; yield: 10\% (42 mg); IR (ATR) $v_{\max } 3071,2961,2921,2850$, $1725(\mathrm{C}=\mathrm{O}), 1652(\mathrm{C}=\mathrm{O}), 1630(\mathrm{C}=\mathrm{C}), 1226,1192,752,692(\operatorname{aromatic} \mathrm{C}-\mathrm{H}) \mathrm{cm}^{-1} ;{ }^{1} \mathrm{H}$ NMR $\left(400 \mathrm{MHz}, \mathrm{CDCl}_{3}\right) \delta(\mathrm{ppm}): 7.72\left(1 \mathrm{H}, \mathrm{d}, J=15.6 \mathrm{~Hz}, \mathrm{H}_{\text {olef. }}\right), 7.53(2 \mathrm{H}, \mathrm{dd}, J=5,2,2 \mathrm{~Hz}$, arom. CH), 7.37 (3H, dd, $J=5.2,2 \mathrm{~Hz}), 6.85\left(1 \mathrm{H}, \mathrm{d}, J=15.6 \mathrm{~Hz}, \mathrm{H}_{\text {olef. }}\right), 3.87-3.58$ (8H, broad), 3.50 $(1 \mathrm{H}, \mathrm{d}, J=15.2 \mathrm{~Hz}, \mathrm{Ha}-3), 2.72(1 \mathrm{H}, \mathrm{d}, J=15.2 \mathrm{~Hz}, \mathrm{Hb}-3), 2.30(2 \mathrm{H}, \mathrm{d}, J=16.0 \mathrm{~Hz}), 2.27$ $(2 \mathrm{H}, \mathrm{s}), 1.63\left(3 \mathrm{H}, \mathrm{s},-\mathrm{CH}_{3}\right), 1.13\left(3 \mathrm{H}, \mathrm{s},-\mathrm{CH}_{3}\right), 1.11\left(3 \mathrm{H}, \mathrm{s},-\mathrm{CH}_{3}\right) ;{ }^{13} \mathrm{C} \mathrm{NMR}\left(100 \mathrm{MHz}, \mathrm{CDCl}_{3}\right)$ $\delta(\mathrm{ppm}): 194.6(\mathrm{C}=\mathrm{O}), 173.0(\mathrm{C}=\mathrm{C}, \mathrm{C}-7 \mathrm{a}), 169.8(\mathrm{C}=\mathrm{O}), 165.7(\mathrm{C}=\mathrm{O}), 143.7(\mathrm{C}=\mathrm{C}), 134.9$, 129.9, 128.9, 127.8, $116.3(\mathrm{C}=\mathrm{C}), 111.4(\mathrm{C}=\mathrm{C}, \mathrm{C}-3 \mathrm{a}), 91.9$, 50.9, 46.0, 37.8, 37.6, 34.3, 28.7 ($\left.\mathrm{CH}_{3}\right), 28.6\left(-\mathrm{CH}_{3}\right), 26.2\left(-\mathrm{CH}_{3}\right)$; HRMS (ESI) (m/z) Calcd for $\mathrm{C}_{25} \mathrm{H}_{30} \mathrm{~N}_{2} \mathrm{O}_{4} 423.22783$ found: $423.22835(\mathrm{M}+\mathrm{H})^{+}$

\section{Trans-3-(4-methacryloylpiperazine-1-carbonyl)-6,6-dimethyl-2-phenyl-3,5,6,7-} tetrahydrobenzofuran- $4(2 \mathrm{H})$-one $(3 \mathrm{~b})$

It was obtained as a yellow oil; yield: 45\% (190 mg); IR (ATR) $v_{\max } 3054,2961,2952,2868$, $1719(\mathrm{C}=\mathrm{O}), 1637(\mathrm{C}=\mathrm{O}), 1610(\mathrm{C}=\mathrm{C}), 1228,1208,760,706(\operatorname{arom} . \mathrm{CH}) \mathrm{cm}^{-1} ;{ }^{1} \mathrm{H}$ NMR $(400$ $\left.\mathrm{MHz}, \mathrm{CDCl}_{3}\right) \delta(\mathrm{ppm}):$ 7.41-7.20 (3H, m, arom. $\left.\mathrm{CH}\right), 7.25-7.23(2 \mathrm{H}, \mathrm{m}$, arom. $\mathrm{CH}), 6.11(1 \mathrm{H}$, d, $J=5.6 \mathrm{~Hz}, \mathrm{H}-2), 5.20\left(1 \mathrm{H}, \mathrm{s}, \mathrm{H}_{\text {olef. }}\right), 5.03\left(1 \mathrm{H}, \mathrm{s}, \mathrm{H}_{\text {olef. }}\right), 4.23(1 \mathrm{H}, \mathrm{d}, J=5.6 \mathrm{~Hz}, \mathrm{H}-3), 4.01-$ $3.30\left(8 \mathrm{H}\right.$, broad), $2.47(2 \mathrm{H}, \mathrm{d}, J=16.0 \mathrm{~Hz}), 2.26(2 \mathrm{H}, \mathrm{d}, J=16.0 \mathrm{~Hz}), 1.94\left(3 \mathrm{H}, \mathrm{s},-\mathrm{CH}_{3}\right), 1.15$ $\left(3 \mathrm{H}, \mathrm{s},-\mathrm{CH}_{3}\right), 1.04\left(3 \mathrm{H}, \mathrm{s},-\mathrm{CH}_{3}\right) ;{ }^{13} \mathrm{C} \mathrm{NMR}\left(100 \mathrm{MHz}, \mathrm{CDCl}_{3}\right) \delta(\mathrm{ppm}): 193.9(\mathrm{C}=\mathrm{O}), 178.1$ $(\mathrm{C}=\mathrm{C}, \mathrm{C}-7 \mathrm{a}), 171.3(\mathrm{C}=\mathrm{O}), 170.3(\mathrm{C}=\mathrm{O}), 140.0(\mathrm{C}=\mathrm{C}), 139.7,129.1,128.9,125.52,116.0$ 
$(\mathrm{C}=\mathrm{C}), 112.1(\mathrm{C}=\mathrm{C}, \mathrm{C}-3 \mathrm{a}), 90.5,51.1,49.9,47.2,44.2,37.9,34.4,28.9\left(-\mathrm{CH}_{3}\right), 28.3\left(-\mathrm{CH}_{3}\right)$, $20.4\left(-\mathrm{CH}_{3}\right)$; HRMS (ESI) (m/z) Calcd for $\mathrm{C}_{25} \mathrm{H}_{30} \mathrm{~N}_{2} \mathrm{O}_{4} 423.22783$ found: $423.22835(\mathrm{M}+\mathrm{H})^{+}$

\section{2,6,6-trimethyl-2-(4-(3-phenylbut-2-enoyl)piperazine-1-carbonyl)-3,5,6,7-}

\section{tetrahydrobenzofuran-4(2H)-one (3c)}

It was obtained as a yellow oil; yield: 45\% (196 mg); IR (ATR) $v_{\max }$ 3058, 2961, 2921, 2850, $1725(\mathrm{C}=\mathrm{O}), 1652(\mathrm{C}=\mathrm{O}), 1630(\mathrm{C}=\mathrm{C}), 1225,1192,752,692(\operatorname{arom} . \mathrm{CH}) \mathrm{cm}^{-1} ;{ }^{1} \mathrm{H}$ NMR $(400$ $\left.\mathrm{MHz}, \mathrm{CDCl}_{3}\right) \delta(\mathrm{ppm}): 7.45(2 \mathrm{H}, \mathrm{d}, \mathrm{J}=8.4,2 \mathrm{~Hz}$, arom. $\mathrm{CH}), 7.39-7.33(3 \mathrm{H}, \mathrm{m}$, arom. $\mathrm{CH})$, $6.26\left(1 \mathrm{H}, \mathrm{s}, \mathrm{H}_{\text {olef }}\right), 3.80-3.54(8 \mathrm{H}$, broad $), 3.49(1 \mathrm{H}, \mathrm{d}, J=15.2 \mathrm{~Hz}, \mathrm{Ha}-3), 2.71(1 \mathrm{H}, \mathrm{d}, J=15.2$ Hz, Hb-3), 2.29 (4H, s), $2.24\left(3 \mathrm{H}, \mathrm{s},-\mathrm{CH}_{3}\right), 1.62\left(3 \mathrm{H}, \mathrm{s},-\mathrm{CH}_{3}\right), 1.12\left(3 \mathrm{H}, \mathrm{s},-\mathrm{CH}_{3}\right), 1.10(3 \mathrm{H}, \mathrm{s}$, $\left.-\mathrm{CH}_{3}\right) ;{ }^{13} \mathrm{C} \mathrm{NMR}\left(100 \mathrm{MHz}, \mathrm{CDCl}_{3}\right) \delta(\mathrm{ppm}): 194.6(\mathrm{C}=\mathrm{O}), 173.0(\mathrm{C}=\mathrm{C}, \mathrm{C}-7 \mathrm{a}), 169.75(\mathrm{C}=\mathrm{O})$, $167.31(\mathrm{C}=\mathrm{O}), 141.4(\mathrm{C}=\mathrm{C}), 128.6,128.5,126.0,118.8,116.1(\mathrm{C}=\mathrm{C}), 111.4(\mathrm{C}=\mathrm{C}, \mathrm{C}-3 \mathrm{a}), 91.9$, 50.9, 46.0, 43.7, 37.8, 34.3, $28.7\left(-\mathrm{CH}_{3}\right), 28.6\left(-\mathrm{CH}_{3}\right), 26.3\left(-\mathrm{CH}_{3}\right), 18.06\left(-\mathrm{CH}_{3}\right) ; \mathrm{HRMS}(\mathrm{ESI})$ $(\mathrm{m} / \mathrm{z})$ Calcd for $\mathrm{C}_{26} \mathrm{H}_{32} \mathrm{~N}_{2} \mathrm{O}_{4} 437.24348$ found $437.24483(\mathrm{M}+\mathrm{H})^{+}$

\section{2-(4-(3,3-diphenylacryloyl)piperazine-1-carbonyl)-2,6,6-trimethyl-3,5,6,7-}

tetrahydrobenzofuran-4(2H)-one $(3 \mathrm{~d})$

It was obtained as a yellow oil; yield: 60\% (300 mg); IR (ATR) $v_{\max } 3058,2961,2925,2872$, $1714(\mathrm{C}=\mathrm{O}), 1632(\mathrm{C}=\mathrm{O}), 1604(\mathrm{C}=\mathrm{C}), 1201,1006,752,701($ arom. $\mathrm{CH}) \mathrm{cm}^{-1} ;{ }^{1} \mathrm{H}$ NMR $(400$ $\left.\mathrm{MHz}, \mathrm{CDCl}_{3}\right) \delta(\mathrm{ppm}):$ 7.37-7.26 (10H, m, arom. $\left.\mathrm{CH}\right), 6.30\left(1 \mathrm{H}, \mathrm{s}, \mathrm{H}_{\mathrm{olef}}\right), 3.48-2.98(8 \mathrm{H}$, broad), $3.39(1 \mathrm{H}, \mathrm{d}, J=15.2 \mathrm{~Hz}, \mathrm{Ha}-3) 2.46(1 \mathrm{H}, \mathrm{d}, J=15.2 \mathrm{~Hz}, \mathrm{Hb}-3), 2.25(2 \mathrm{H}, \mathrm{d}, J=16.4$ $\mathrm{Hz}), 2.20(2 \mathrm{H}, \mathrm{d}, J=16.4 \mathrm{~Hz}), 1.53\left(3 \mathrm{H}, \mathrm{s},-\mathrm{CH}_{3}\right), 1.11\left(3 \mathrm{H}, \mathrm{s},-\mathrm{CH}_{3}\right), 1.08\left(3 \mathrm{H}, \mathrm{s},-\mathrm{CH}_{3}\right) ;{ }^{13} \mathrm{C}$ NMR (100 MHz, $\left.\mathrm{CDCl}_{3}\right) \delta(\mathrm{ppm}): 194.5(\mathrm{C}=\mathrm{O}), 173.0(\mathrm{C}=\mathrm{C}, \mathrm{C}-7 \mathrm{a}) 169.4(\mathrm{C}=\mathrm{O}), 167.34$ $(\mathrm{C}=\mathrm{O}), 147.9(\mathrm{C}=\mathrm{C}), 140.5,138.7,129.5,128.8,128.4,128.4,128.0,111.4(\mathrm{C}=\mathrm{C}), 110.0(\mathrm{C}=\mathrm{C}$, C-3a), 91.7, 50.88, 46.1, 45.5, 42.9, 41.1, 37.7, 37.5, 34.2, $28.72\left(-\mathrm{CH}_{3}\right), 28.6\left(-\mathrm{CH}_{3}\right), 26.16(-$ $\left.\mathrm{CH}_{3}\right)$; HRMS (ESI) (m/z) Calcd for $\mathrm{C}_{31} \mathrm{H}_{34} \mathrm{~N}_{2} \mathrm{O}_{4} 499.25913$ found $499.26110(\mathrm{M}+\mathrm{H})^{+}$ 


\section{Trans-3-(4-methacryloylpiperazine-1-carbonyl)-6,6-dimethyl-2-styryl-3,5,6,7-}

tetrahydrobenzofuran-4(2H)-one (3e)

It was obtained as a yellow oil; yield: $20 \%$ (89 mg); IR (ATR) $v_{\max } 3067,2965,2925,2854$, $1734(\mathrm{C}=\mathrm{O}), 1646(\mathrm{C}=\mathrm{O}), 1626(\mathrm{C}=\mathrm{C}), 1191,1090,754,695$ (arom. $\mathrm{CH}) \mathrm{cm}^{-1} ;{ }^{1} \mathrm{H}$ NMR $(400$ $\left.\mathrm{MHz}, \mathrm{CDCl}_{3}\right) \delta(\mathrm{ppm}): 7.31(5 \mathrm{H}, \mathrm{m}, \operatorname{arom} . \mathrm{CH}), 6.66\left(1 \mathrm{H}, \mathrm{d}, J=16.0 \mathrm{~Hz}, \mathrm{H}_{\text {olef }}\right), 6.20(1 \mathrm{H}, \mathrm{dd}$, $\left.J=16.0,7.6 \mathrm{~Hz}, \mathrm{H}_{\mathrm{olef}}\right), 5.74(1 \mathrm{H}, \mathrm{dd}, J=7.6,6.4 \mathrm{~Hz}, \mathrm{H}-2), 5.22(1 \mathrm{H}, \mathrm{s}), 5.05(1 \mathrm{H}, \mathrm{s}), 3.71(1 \mathrm{H}$, $\mathrm{d}, J=6.4 \mathrm{~Hz}, \mathrm{H}-3), 3.98-3.26(8 \mathrm{H}$, broad $), 2.41(2 \mathrm{H}, \mathrm{d}, J=17.0 \mathrm{~Hz}) 2.20(2 \mathrm{H}, \mathrm{d}, J=16.4 \mathrm{~Hz})$ , $1.96\left(3 \mathrm{H}, \mathrm{s},-\mathrm{CH}_{3}\right), 1.14\left(3 \mathrm{H}, \mathrm{s},-\mathrm{CH}_{3}\right) 1.12\left(3 \mathrm{H}, \mathrm{s},-\mathrm{CH}_{3}\right) ;{ }^{13} \mathrm{C}-\mathrm{NMR}\left(100 \mathrm{MHz}, \mathrm{CDCl}_{3}\right), \delta$ (ppm): $193.9(\mathrm{C}=\mathrm{O}), 177.7(\mathrm{C}=\mathrm{C}, \mathrm{C}-7 \mathrm{a}), 171.3(\mathrm{C}=\mathrm{O}), 170.2(\mathrm{C}=\mathrm{O}), 140.0(\mathrm{C}=\mathrm{C}), 135.3$, 134.1, $128.8(\mathrm{C}=\mathrm{C}), 128.7,128.6,126.8,125.4(\mathrm{C}=\mathrm{C}), 115.9(\mathrm{C}=\mathrm{C}), 112.1(\mathrm{C}=\mathrm{C}, \mathrm{C}-3 \mathrm{a}), 90.23$, 51.06, 47.6, 46.4, 42.3, 37.9, 34.3, $28.7\left(-\mathrm{CH}_{3}\right), 28.4\left(-\mathrm{CH}_{3}\right), 20.4\left(-\mathrm{CH}_{3}\right) ; \mathrm{HRMS}(\mathrm{ESI})(\mathrm{m} / \mathrm{z})$ Calcd for $\mathrm{C}_{27} \mathrm{H}_{32} \mathrm{~N}_{2} \mathrm{O}_{4} 449.24348$ found $449.24527(\mathrm{M}+\mathrm{H})^{+}$

\section{2,6,6-trimethyl-2-(4-(3-(thiophen-2-yl)but-2-enoyl)piperazine-1-carbonyl)-3,5,6,7- tetrahydrobenzofuran-4(2H)-one (3f)}

It was obtained as a yellow oil; yield: 10\% (44 mg); IR (ATR) $v_{\max } 3071,2965,2921,2863$, $1716(\mathrm{C}=\mathrm{O}), 1650(\mathrm{C}=\mathrm{O}), 1621(\mathrm{C}=\mathrm{C}), 1194,1017,759,692(\operatorname{arom} . \mathrm{CH}) \mathrm{cm}^{-1} ;{ }^{1} \mathrm{H}$ NMR $(400$ $\left.\mathrm{MHz}, \mathrm{CDCl}_{3}\right) \delta(\mathrm{ppm}): 7.28(1 \mathrm{H}, \mathrm{d}, J=0.8 \mathrm{~Hz}$, arom. $\mathrm{CH}), 7.21(1 \mathrm{H}, \mathrm{dd}, J=4.0,0.8 \mathrm{~Hz}$, arom. $\mathrm{CH}), 7.03(1 \mathrm{H}, \mathrm{dd}, J=6.0,4.0 \mathrm{~Hz}$, arom. $\mathrm{CH}), 6.38\left(1 \mathrm{H}, \mathrm{s}, \mathrm{H}_{\text {olef }}\right), 3.79-3.55$ (8H, broad), 3.50 $(1 \mathrm{H}, \mathrm{d}, J=15.2 \mathrm{~Hz}, \mathrm{Ha}-3), 2.72(1 \mathrm{H}, \mathrm{d}, J=15.2 \mathrm{~Hz}, \mathrm{Hb}-3), 2.31(2 \mathrm{H}, \mathrm{d}, J=16.0 \mathrm{~Hz}), 2.29$ $(2 \mathrm{H}, J=16.0 \mathrm{~Hz}), 2.25\left(3 \mathrm{H}, \mathrm{s},-\mathrm{CH}_{3}\right), 1.62\left(3 \mathrm{H}, \mathrm{s},-\mathrm{CH}_{3}\right), 1.12\left(3 \mathrm{H}, \mathrm{s},-\mathrm{CH}_{3}\right), 1.11(3 \mathrm{H}, \mathrm{s},-$ $\left.\mathrm{CH}_{3}\right) ;{ }^{13} \mathrm{C}-\mathrm{NMR}\left(100 \mathrm{MHz}, \mathrm{CDCl}_{3}\right), \delta(\mathrm{ppm}): 194.6(\mathrm{C}=\mathrm{O}), 172.9(\mathrm{C}=\mathrm{C}, \mathrm{C}-7 \mathrm{a}), 171.9(\mathrm{C}=\mathrm{O})$, $169.7(\mathrm{C}=\mathrm{O}), 145.1(\mathrm{C}=\mathrm{C}), 127.8,125.8,125.6,116.1(\mathrm{C}=\mathrm{C}), 111.5(\mathrm{C}=\mathrm{C}, \mathrm{C}-3 \mathrm{a}), 91.9,50.9$, 46.3, 45.8, 37.8, 37.5, 34.2, $29.6\left(-\mathrm{CH}_{3}\right), 28.6\left(-\mathrm{CH}_{3}\right), 26.2\left(-\mathrm{CH}_{3}\right), 17.8\left(-\mathrm{CH}_{3}\right)$; HRMS (ESI) $(\mathrm{m} / \mathrm{z})$ Calcd for $\mathrm{C}_{24} \mathrm{H}_{30} \mathrm{~N}_{2} \mathrm{O}_{4} \mathrm{~S} 443.19990$ found $443.20162(\mathrm{M}+\mathrm{H})^{+}$ 


\section{3-(4-methacryloylpiperazine-1-carbonyl)-2,6,6-trimethyl-2-(thiophen-2-yl)-3,5,6,7-}

tetrahydrobenzofuran-4(2H)-one $(3 \mathrm{~g})$

It was obtained as a yellow oil; yield: 40\% (177 mg); IR (ATR) $v_{\max } 3071,2956,2921,2859$, $1736(\mathrm{C}=\mathrm{O}), 1643(\mathrm{C}=\mathrm{O}), 1610(\mathrm{C}=\mathrm{C}), 1194,1024,755,700(\operatorname{arom~CH}) \mathrm{cm}^{-1} ;{ }^{1} \mathrm{H}$ NMR $(400$ $\left.\mathrm{MHz}, \mathrm{CDCl}_{3}\right) \delta(\mathrm{ppm}): 7.28(1 \mathrm{H}, \mathrm{d}, J=4.4 \mathrm{~Hz}$, arom. $\mathrm{CH}), 7.00-6.97$ (2H, m, arom. $\left.\mathrm{CH}\right), 5.22$ (1H, s, $\left.\mathrm{H}_{\text {olef }}\right), 5.05$ (1H, s, $\left.\mathrm{H}_{\text {olef }}\right), 4.46$ (1H, s, H-3), 3.60-3.52 (8H, broad), 2.40 (2H, d, J= 16.4 $\mathrm{Hz}), 2.27(2 \mathrm{H}, \mathrm{d}, J=16.4 \mathrm{~Hz}), 1.95\left(3 \mathrm{H}, \mathrm{s},-\mathrm{CH}_{3}\right), 1.83\left(3 \mathrm{H}, \mathrm{s},-\mathrm{CH}_{3}\right), 1.46\left(3 \mathrm{H}, \mathrm{s},-\mathrm{CH}_{3}\right), 1.21$ $\left(3 \mathrm{H}, \mathrm{s},-\mathrm{CH}_{3}\right) ;{ }^{13} \mathrm{C}-\mathrm{NMR}\left(100 \mathrm{MHz}, \mathrm{CDCl}_{3}\right), \delta(\mathrm{ppm}): 194.1(\mathrm{C}=\mathrm{O}), 175.6(\mathrm{C}=\mathrm{C}, \mathrm{C}-7 \mathrm{a}), 171.3$ $(\mathrm{C}=\mathrm{O}), 167.94(\mathrm{C}=\mathrm{O}), 148.9,139.9(\mathrm{C}=\mathrm{C}), 127.0,125.4,123.2,116.1(\mathrm{C}=\mathrm{C}), 112.7(\mathrm{C}=\mathrm{C}, \mathrm{C}-$ 3a), 90.8, 53.0, 50.73, 46.28, 42.66, 37.7, 34.6, $28.7\left(-\mathrm{CH}_{3}\right), 28.4\left(-\mathrm{CH}_{3}\right), 23.8\left(-\mathrm{CH}_{3}\right), 20.45(-$ $\left.\mathrm{CH}_{3}\right)$; HRMS (ESI) (m/z) Calcd for $\mathrm{C}_{24} \mathrm{H}_{30} \mathrm{~N}_{2} \mathrm{O}_{4} \mathrm{~S} 443.19990$ found $443.20162(\mathrm{M}+\mathrm{H})^{+}$

\section{1-(4-(4-acetyl-2,5-dimethyl-2,3-dihydrofuran-2-carbonyl)piperazin-1-yl)-3-phenylprop-}

\section{2-en-1-one (3h)}

It was obtained as a yellow oil; yield: 45\% (172 mg); IR (ATR) $v_{\max } 3076,2965,2912,2845$, $1714(\mathrm{C}=\mathrm{O}), 1632(\mathrm{C}=\mathrm{O}), 1602(\mathrm{C}=\mathrm{C}), 1230,1022,756,708(\operatorname{arom} . \mathrm{CH}) \mathrm{cm}^{-1} ;{ }^{1} \mathrm{H}$ NMR $(400$ $\left.\mathrm{MHz}, \mathrm{CDCl}_{3}\right) \delta(\mathrm{ppm}): 7.72\left(1 \mathrm{H}, \mathrm{d}, J=15.2 \mathrm{~Hz}, \mathrm{H}_{\text {olef }}\right), 7.54-7.52(2 \mathrm{H}, \mathrm{m}$, arom. $\mathrm{CH}), 7.41-$ $7.36\left(3 \mathrm{H}, \mathrm{m}\right.$, arom. CH), $7.86\left(1 \mathrm{H}, \mathrm{d}, J=15.2 \mathrm{~Hz}, \mathrm{H}_{\text {olef }}\right), 3.78(1 \mathrm{H}, \mathrm{d}, J=14.8 \mathrm{~Hz}, \mathrm{H}-3), 3.98-$ $3.56\left(8 \mathrm{H}\right.$, broad), $2.79(1 \mathrm{H}, \mathrm{d}, J=14.8 \mathrm{~Hz}, \mathrm{H}-3), 2.23\left(3 \mathrm{H}, \mathrm{s},-\mathrm{CH}_{3}\right), 2.20\left(3 \mathrm{H}, \mathrm{s},-\mathrm{CH}_{3}\right) 1.62$ (3H, s, $\left.-\mathrm{CH}_{3}\right) ;{ }^{13} \mathrm{C}-\mathrm{NMR}\left(100 \mathrm{MHz}, \mathrm{CDCl}_{3}\right), \delta(\mathrm{ppm}): 191.0(\mathrm{C}=\mathrm{O}), 177.5(\mathrm{C}=\mathrm{C}, \mathrm{C}-5), 169.7$ $(\mathrm{C}=\mathrm{O}), 165.5(\mathrm{C}=\mathrm{O}), 143.7(\mathrm{C}=\mathrm{C}), 134.9,129.9,128.8,127.8,116.3(\mathrm{C}=\mathrm{C}), 114.2(\mathrm{C}=\mathrm{C}, \mathrm{C}-$ 4), 88.6, 46.5, 44.3, 42.0, $29.6\left(-\mathrm{CH}_{3}\right), 26.1\left(-\mathrm{CH}_{3}\right), 24.5\left(-\mathrm{CH}_{3}\right), 14.84\left(-\mathrm{CH}_{3}\right) ;$ HRMS (ESI) (m/z) Calcd for $\mathrm{C}_{22} \mathrm{H}_{26} \mathrm{~N}_{2} \mathrm{O}_{4} 383.19653$ found $383.19745(\mathrm{M}+\mathrm{H})^{+}$

\section{1-(4-(4-acetyl-2,5-dimethyl-2,3-dihydrofuran-2-carbonyl)piperazin-1-yl)-3,3-}

\section{diphenylprop-2-en-1-one (3i)}

It was obtained as a yellow oil; yield: 30\% (137 mg); IR (ATR) $v_{\max } 3058,2978,2925,2863$, $1725(\mathrm{C}=\mathrm{O}), 1630(\mathrm{C}=\mathrm{O}), 1604(\mathrm{C}=\mathrm{C}), 1239,1026,759,701$ (arom. $\mathrm{CH}) \mathrm{cm}^{-1} ;{ }^{1} \mathrm{H}$ NMR $(400$ 
$\left.\mathrm{MHz}, \mathrm{CDCl}_{3}\right) \delta(\mathrm{ppm}): 7.33(10 \mathrm{H}, \mathrm{m}, \operatorname{arom~CH}), 6.30\left(1 \mathrm{H}, \mathrm{s}, \mathrm{H}_{\text {olef }}\right), 3.69(1 \mathrm{H}, \mathrm{d}, J=15.2 \mathrm{~Hz}$, H-3), 3.79-2.78 (8H, broad), $2.71\left(1 \mathrm{H}, \mathrm{d}, J=15.2 \mathrm{~Hz}, \mathrm{H}_{\text {olef }}\right), 2.17\left(6 \mathrm{H}, \mathrm{s},-\mathrm{CH}_{3}\right) 1.63(3 \mathrm{H}, \mathrm{s},-$ $\left.\mathrm{CH}_{3}\right) ;{ }^{13} \mathrm{C}-\mathrm{NMR}\left(100 \mathrm{MHz}, \mathrm{CDCl}_{3}\right), \delta(\mathrm{ppm}): 192.0(\mathrm{C}=\mathrm{O}), 176.5(\mathrm{C}=\mathrm{C}, \mathrm{C}-5), 171.7(\mathrm{C}=\mathrm{O})$, $167.5(\mathrm{C}=\mathrm{O}), 140.5,138.7,129.5,128.8,128.8,128.4,128.4,128.1,120.1,117.5(\mathrm{C}=\mathrm{C}), 114.8$ $(\mathrm{C}=\mathrm{C}, \mathrm{C}-4), 88.5,45.8,41.9,27.0,25.3\left(-\mathrm{CH}_{3}\right), 23.4\left(-\mathrm{CH}_{3}\right), 12.2\left(-\mathrm{CH}_{3}\right) ; \mathrm{HRMS}(\mathrm{ESI})(\mathrm{m} / \mathrm{z})$ Calcd for $\mathrm{C}_{28} \mathrm{H}_{30} \mathrm{~N}_{2} \mathrm{O}_{4} 459.22783$ found $459.22902(\mathrm{M}+\mathrm{H})^{+}$

Ethyl 5-(4-cinnamoylpiperazine-1-carbonyl)-2,5-dimethyl-4,5-dihydrofuran-3carboxylate $(\mathbf{3 j})$

It was obtained as a yellow oil; yield: $13 \%$ (51 mg); IR (ATR) $v_{\max } 3067,2969,2930,2872$, $1736(\mathrm{C}=\mathrm{O}), 1643(\mathrm{C}=\mathrm{O}), 1608(\mathrm{C}=\mathrm{C}), 1194,1090,761,701($ arom. $\mathrm{CH}) \mathrm{cm}^{-1} ;{ }^{1} \mathrm{H}$ NMR $(400$ $\left.\mathrm{MHz}, \mathrm{CDCl}_{3}\right) \delta(\mathrm{ppm}): 7.71\left(1 \mathrm{H}, \mathrm{d}, J=15.2 \mathrm{~Hz}, \mathrm{H}_{\mathrm{olef}}\right), 7.53(2 \mathrm{H}, \mathrm{dd}, J=5.2,2 \mathrm{~Hz}$ arom. $\mathrm{CH})$, 7.40-7.36 (3H, m, arom. CH), $6.87\left(1 \mathrm{H}, \mathrm{d}, J=15.2 \mathrm{~Hz}, \mathrm{H}_{\text {olef }}\right), 4.17(2 \mathrm{H}, \mathrm{q}, J=7.2 \mathrm{~Hz},-$ $\left.\mathrm{OC}_{2} \mathrm{CH}_{3}\right), 3.92-3.55(8 \mathrm{H}$, broad), $3.61(1 \mathrm{H}, \mathrm{d}, J=15.2 \mathrm{~Hz}, \mathrm{Ha}-4) 2.74(1 \mathrm{H}, \mathrm{d}, J=15.2 \mathrm{~Hz}$, $\mathrm{Hb}-4), 2.21\left(3 \mathrm{H}, \mathrm{s},-\mathrm{CH}_{3}\right), 1.59\left(3 \mathrm{H}, \mathrm{s},-\mathrm{CH}_{3}\right) 1.27\left(3 \mathrm{H}, \mathrm{t}, J=7.2 \mathrm{~Hz},-\mathrm{OCH}_{2} \mathrm{CH}_{3}\right) ;{ }^{13} \mathrm{C}-\mathrm{NMR}$ $\left(100 \mathrm{MHz}, \mathrm{CDCl}_{3}\right), \delta(\mathrm{ppm}): 170.5(\mathrm{C}=\mathrm{C}, \mathrm{C}-2), 165.7(\mathrm{C}=\mathrm{O}), 165.5(\mathrm{C}=\mathrm{O}), 164.5(\mathrm{C}=\mathrm{O}), 143.5$ $(\mathrm{C}=\mathrm{C}), 134.9,129.8,129.0,128.8,127.8,125.4,116.4(\mathrm{C}=\mathrm{C}), 102.4(\mathrm{C}=\mathrm{C}, \mathrm{C}-3), 88.3,59.7$, 46.2, 43.5, 42.2, 41.2, $26.0\left(-\mathrm{CH}_{3}\right), 14.3\left(-\mathrm{CH}_{3}\right), 14.1\left(-\mathrm{CH}_{3}\right)$; HRMS (ESI) (m/z) Calcd for $\mathrm{C}_{23} \mathrm{H}_{28} \mathrm{~N}_{2} \mathrm{O}_{5} 413.20710$ found $413.20625(\mathrm{M}+\mathrm{H})^{+}$

\section{Trans-Ethyl} 4-(4-methacryloylpiperazine-1-carbonyl)-2-methyl-5-phenyl-4,5dihydrofuran-3-carboxylate $(3 \mathbf{k})$

It was obtained as a yellow oil; yield: 25\% (105 mg); IR (ATR) $v_{\max } 3026,2966,2930,2870$, $1740(\mathrm{C}=\mathrm{O}), 1638(\mathrm{C}=\mathrm{O}), 1610(\mathrm{C}=\mathrm{C}), 1200,1025,750,700(\operatorname{arom~CH}) \mathrm{cm}^{-1} ;{ }^{1} \mathrm{H}$ NMR $(400$ $\left.\mathrm{MHz}, \mathrm{CDCl}_{3}\right) \delta(\mathrm{ppm}):$ 7.42-7.33 (3H, m, arom. $\left.\mathrm{CH}\right)$, 7.29-7.26 (2H, m, arom. $\left.\mathrm{CH}\right), 5.67(1 \mathrm{H}$, d, $J=7.2 \mathrm{~Hz}, \mathrm{H}-5), 5.21\left(1 \mathrm{H}, \mathrm{s}, \mathrm{H}_{\text {olef }}\right), 5.02\left(1 \mathrm{H}, \mathrm{s}, \mathrm{H}_{\mathrm{olef}}\right), 4.35(1 \mathrm{H}, \mathrm{d}, J=7.2 \mathrm{~Hz}, \mathrm{H}-4), 4.15$ $\left(2 \mathrm{H}, \mathrm{q}, J=7.2 \mathrm{~Hz},-\mathrm{OC}_{2} \mathrm{CH}_{3}\right), 3.80-3.30(8 \mathrm{H}, \mathrm{broad}), 2.35\left(3 \mathrm{H}, \mathrm{s},-\mathrm{CH}_{3}\right), 1.94\left(3 \mathrm{H}, \mathrm{s},-\mathrm{CH}_{3}\right)$ 
$1.26\left(3 \mathrm{H}, \mathrm{t}, J=7.2 \mathrm{~Hz},-\mathrm{OCH}_{2} \underline{\mathrm{C}}_{3}\right) ;{ }^{13} \mathrm{C}-\mathrm{NMR}\left(100 \mathrm{MHz}, \mathrm{CDCl}_{3}\right), \delta(\mathrm{ppm}): 171.7(\mathrm{C}=\mathrm{C}, \mathrm{C}-$ 2), $171.2(\mathrm{C}=\mathrm{O}), 169.5(\mathrm{C}=\mathrm{O}), 165.0(\mathrm{C}=\mathrm{O}), 139.9(\mathrm{C}=\mathrm{C}), 139.7,129.0,128.8,127.8,125.4$, $116.0(\mathrm{C}=\mathrm{C}), 103.65(\mathrm{C}=\mathrm{C}, \mathrm{C}-3), 87.3,59.9,46.1,42.0,20.4\left(-\mathrm{CH}_{3}\right), 14.56\left(-\mathrm{CH}_{3}\right), 14.43(-$ $\left.\mathrm{CH}_{3}\right)$; HRMS (ESI) (m/z) Calcd for $\mathrm{C}_{23} \mathrm{H}_{28} \mathrm{~N}_{2} \mathrm{O}_{5} 413.20710$ found $413.20919(\mathrm{M}+\mathrm{H})^{+}$

Ethyl-2,5-dimethyl-5-(4-(3-phenylbut-2-enoyl)piperazine-1-carbonyl)-4,5-dihydrofuran3-carboxylate (31)

It was obtained as a yellow oil; yield: 40\% (170,8 mg); IR (ATR) $v_{\max } 3054,2961,2916,2868$, $1732(\mathrm{C}=\mathrm{O}), 1696(\mathrm{C}=\mathrm{O}), 1617(\mathrm{C}=\mathrm{C}), 1228,1097,750,706$ (arom. $\mathrm{CH}) \mathrm{cm}^{-1} ;{ }^{1} \mathrm{H}$ NMR $(400$ $\left.\mathrm{MHz}, \mathrm{CDCl}_{3}\right) \delta(\mathrm{ppm}): 7.45(2 \mathrm{H}, \mathrm{dd}, \mathrm{J}=8.4,1.6 \mathrm{~Hz}$, arom. $\mathrm{CH}), 7.36(3 \mathrm{H}, \mathrm{m}), 6.24(1 \mathrm{H}, \mathrm{s}$, $\left.\mathrm{H}_{\text {olef }}\right), 4.16\left(2 \mathrm{H}, \mathrm{q}, J=7.2 \mathrm{~Hz},-\mathrm{OC}_{2} \mathrm{CH}_{3}\right), 3.92-3.51(8 \mathrm{H}$, broad $), 3.61(1 \mathrm{H}, \mathrm{d}, J=15.2 \mathrm{~Hz}$, Ha-4), $2.73(1 \mathrm{H}, \mathrm{d}, J=15.2 \mathrm{~Hz}, \mathrm{Hb}-4), 2.29\left(3 \mathrm{H}, \mathrm{s},-\mathrm{CH}_{3}\right), 2.19\left(3 \mathrm{H}, \mathrm{s},-\mathrm{CH}_{3}\right), 1.58(3 \mathrm{H}, \mathrm{s},-$ $\left.\mathrm{CH}_{3}\right), 1.27\left(3 \mathrm{H}, \mathrm{t}, J=7.2 \mathrm{~Hz},-\mathrm{OCH}_{2} \mathrm{CH}_{3}\right) ;{ }^{13} \mathrm{C}-\mathrm{NMR}\left(100 \mathrm{MHz}, \mathrm{CDCl}_{3}\right), \delta(\mathrm{ppm}): 171.5(\mathrm{C}=\mathrm{C}$, $\mathrm{C}-2), 170.6(\mathrm{C}=\mathrm{O}), 165.6(\mathrm{C}=\mathrm{O}), 164.5(\mathrm{C}=\mathrm{O}), 141.4(\mathrm{C}=\mathrm{C}), 128.5,128.5,125.9,118.8(\mathrm{C}=\mathrm{C})$, $102.3(\mathrm{C}=\mathrm{C}, \mathrm{C}-3), 88.3,59.7,46.3,43.4,41.2,26.0\left(-\mathrm{CH}_{3}\right), 18.03\left(-\mathrm{CH}_{3}\right), 14.37\left(-\mathrm{CH}_{3}\right), 14.07$ $\left(-\mathrm{CH}_{3}\right) ; \mathrm{HRMS}(\mathrm{ESI})(\mathrm{m} / \mathrm{z})$ Calcd for $\mathrm{C}_{24} \mathrm{H}_{30} \mathrm{~N}_{2} \mathrm{O}_{5} 427.22275$ found $427.22406(\mathrm{M}+\mathrm{H})^{+}$

Ethyl 5-(4-(3,3-diphenylacryloyl)piperazine-1-carbonyl)-2,5-dimethyl-4,5-dihydrofuran3-carboxylate (3m)

It was obtained as a yellow oil; yield: 50\% (244 mg); IR (ATR) $v_{\max } 3054,2961,2921,2863$, $1730(\mathrm{C}=\mathrm{O}), 1650(\mathrm{C}=\mathrm{O}), 1620(\mathrm{C}=\mathrm{C}), 1228,1060,760,703($ arom. $\mathrm{CH}) \mathrm{cm}^{-1} ;{ }^{1} \mathrm{H}$ NMR $(400$ $\left.\mathrm{MHz}, \mathrm{CDCl}_{3}\right) \delta(\mathrm{ppm}):$ 7.37-7.27 (10H, m, arom. $\left.\mathrm{CH}\right), 6.30\left(1 \mathrm{H}, \mathrm{s}, \mathrm{H}_{\text {olef }}\right), 4.15(2 \mathrm{H}, \mathrm{q}, J=6.8$ $\left.\mathrm{Hz},-\mathrm{OC} \underline{H}_{2} \mathrm{CH}_{3}\right), 3.52(1 \mathrm{H}, \mathrm{d}, J=15.2 \mathrm{~Hz}, \mathrm{H}-4), 3.75-2.76(8 \mathrm{H}$, broad $), 2.66(1 \mathrm{H}, \mathrm{d}, J=15.2$ $\mathrm{Hz}, \mathrm{H}-4), 2.15\left(3 \mathrm{H}, \mathrm{s},-\mathrm{CH}_{3}\right), 1.50\left(3 \mathrm{H}, \mathrm{s},-\mathrm{CH}_{3}\right), 1.25\left(3 \mathrm{H}, \mathrm{t}, J=6.8 \mathrm{~Hz},-\mathrm{OCH}_{2} \mathrm{CH}_{3}\right) ;{ }^{13} \mathrm{C}-\mathrm{NMR}$ $\left(100 \mathrm{MHz}, \mathrm{CDCl}_{3}\right), \delta(\mathrm{ppm}): 172.8(\mathrm{C}=\mathrm{C}, \mathrm{C}-2), 170.3(\mathrm{C}=\mathrm{O}), 167.2(\mathrm{C}=\mathrm{O}), 165.6(\mathrm{C}=\mathrm{O}), 140.5$ $129.5,128.8,128.7,128.4,128.4,128.1,120.0,110.0(\mathrm{C}=\mathrm{C}), 102.3(\mathrm{C}=\mathrm{C}, \mathrm{C}-3), 88.2,59.7$, 
46.1, 45.6, 41.1, $25.9\left(-\mathrm{CH}_{3}\right), 14.3\left(-\mathrm{CH}_{3}\right), 14.0\left(-\mathrm{CH}_{3}\right)$; HRMS (ESI) (m/z) Calcd for $\mathrm{C}_{29} \mathrm{H}_{32} \mathrm{~N}_{2} \mathrm{O}_{5} 489.23840$ found $489.23854(\mathrm{M}+\mathrm{H})^{+}$

Ethyl-2,5-dimethyl-5-(4-(3-(thiophen-2-yl)acryloyl)piperazine-1-carbonyl)-4,5dihydrofuran-3-carboxylate (3n)

It was obtained as a yellow oil; yield: $20 \%$ (84 mg); IR (ATR) $v_{\max } 3080,2961,2916,2850$, 2954, $1734(\mathrm{C}=\mathrm{O}), 1694(\mathrm{C}=\mathrm{O}), 1631(\mathrm{C}=\mathrm{C}), 1236,1090,760,701(\operatorname{arom} . \mathrm{CH}) \mathrm{cm}^{-1} ;{ }^{1} \mathrm{H} \mathrm{NMR}$ $\left(400 \mathrm{MHz}, \mathrm{CDCl}_{3}\right) \delta(\mathrm{ppm}): 7.84\left(1 \mathrm{H}, \mathrm{d}, J=14.8 \mathrm{~Hz}, \mathrm{H}_{\text {olef }}\right), 7.34(1 \mathrm{H}, \mathrm{d}, J=4.4 \mathrm{~Hz}$, arom. $\mathrm{CH}), 7.23(1 \mathrm{H}, \mathrm{d}, J=3.2 \mathrm{~Hz}$, arom. $\mathrm{CH}), 7.05(1 \mathrm{H}, \mathrm{d}, J=3.2 \mathrm{~Hz}$, arom. $\mathrm{CH}) 6.65(1 \mathrm{H}, \mathrm{d}, J=$ $\left.14.8 \mathrm{~Hz}, \mathrm{H}_{\text {olef }}\right), 4.17\left(2 \mathrm{H}, \mathrm{q}, J=7.2 \mathrm{~Hz},-\mathrm{OC}_{2} \mathrm{CH}_{3}\right), 3.92-3.38(8 \mathrm{H}$, broad $) 3.63(1 \mathrm{H}, \mathrm{d}, J=$ 15.2 Hz, Ha-4), $2.73(1 \mathrm{H}, \mathrm{d}, J=15.2 \mathrm{~Hz}, \mathrm{Hb}-4), 2.20\left(3 \mathrm{H}, \mathrm{s},-\mathrm{CH}_{3}\right), 1.59\left(3 \mathrm{H}, \mathrm{s},-\mathrm{CH}_{3}\right), 1.28$ $\left(3 \mathrm{H}, \mathrm{t}, J=7.2 \mathrm{~Hz},-\mathrm{OCH}_{2} \mathrm{CH}_{3}\right) ;{ }^{13} \mathrm{C}-\mathrm{NMR}\left(100 \mathrm{MHz}, \mathrm{CDCl}_{3}\right), \delta(\mathrm{ppm}): 171.4(\mathrm{C}=\mathrm{C}, \mathrm{C}-2)$, $170.3(\mathrm{C}=\mathrm{O}), 167.3(\mathrm{C}=\mathrm{O}), 165.6(\mathrm{C}=\mathrm{O}), 136.4,130.6,128.0,127.5,114.9(\mathrm{C}=\mathrm{C}), 110.0(\mathrm{C}=\mathrm{C}$, C-3), 88.3, 59.7, 45.4, 42.3, 41.2, $29.68\left(-\mathrm{CH}_{3}\right), 14.37\left(-\mathrm{CH}_{3}\right), 14.09\left(-\mathrm{CH}_{3}\right) ; \mathrm{HRMS}(\mathrm{ESI})(\mathrm{m} / \mathrm{z})$ Calcd for $\mathrm{C}_{21} \mathrm{H}_{26} \mathrm{~N}_{2} \mathrm{O}_{5} \mathrm{~S} 419.16352$ found $419.16496(\mathrm{M}+\mathrm{H})^{+}$

Trans-Ethyl 4-(4-methacryloylpiperazine-1-carbonyl)-2-methyl-5-(thiophen-2-yl)-4,5dihydrofuran-3-carboxylate (3o)

It was obtained as a yellow oil; yield: 30\% (125 mg); IR (ATR) $v_{\max } 3085,2974,2921,2863$, $1732(\mathrm{C}=\mathrm{O}), 1692(\mathrm{C}=\mathrm{O}), 1621(\mathrm{C}=\mathrm{C}), 1194,1080,756,703(\operatorname{arom} . \mathrm{CH}) \mathrm{cm}^{-1} ;{ }^{1} \mathrm{H}$ NMR $(400$ $\left.\mathrm{MHz}, \mathrm{CDCl}_{3}\right) \delta(\mathrm{ppm}): 7.33(1 \mathrm{H}, \mathrm{dd}, J=4.8,1.2 \mathrm{~Hz}$, arom. $\mathrm{CH}), 7.17(1 \mathrm{H}, \mathrm{dd}, J=4.8,1.2$ Hz, arom. CH), $7.00(1 \mathrm{H}, \mathrm{dd}, J=4.8,1.2 \mathrm{~Hz}$, arom. $\mathrm{CH}), 5.90(1 \mathrm{H}, \mathrm{d}, J=7.2 \mathrm{~Hz}, \mathrm{H}-5), 5.22$ (1H, s, $\left.\mathrm{H}_{\text {olef }}\right), 5.03\left(1 \mathrm{H}, \mathrm{s}, \mathrm{H}_{\text {olef }}\right), 4.50(1 \mathrm{H}, \mathrm{d}, J=7.2 \mathrm{~Hz}, \mathrm{H}-4), 4.14(2 \mathrm{H}, \mathrm{q}, J=7.2 \mathrm{~Hz},-$ OC $\left.\underline{\mathrm{H}}_{2} \mathrm{CH}_{3}\right), 3.73-3.50(8 \mathrm{H}$, broad $), 2.29\left(3 \mathrm{H}, \mathrm{s},-\mathrm{CH}_{3}\right), 1.94\left(3 \mathrm{H}, \mathrm{s},-\mathrm{CH}_{3}\right), 1.28(3 \mathrm{H}, \mathrm{t}, J=7.2$ $\left.\mathrm{Hz},-\mathrm{OCH}_{2} \underline{\mathrm{C}}_{3}\right) ;{ }^{13} \mathrm{C}-\mathrm{NMR}\left(100 \mathrm{MHz}, \mathrm{CDCl}_{3}\right), \delta(\mathrm{ppm}): 171.3(\mathrm{C}=\mathrm{C}, \mathrm{C}-2), 171.1(\mathrm{C}=\mathrm{O}), 168.8$ $(\mathrm{C}=\mathrm{O}), 164.8(\mathrm{C}=\mathrm{O}), 142.2,139.9(\mathrm{C}=\mathrm{C}), 127.1,126.3,126.05,116.0(\mathrm{C}=\mathrm{C}), 103.7(\mathrm{C}=\mathrm{C}, \mathrm{C}-$ 
3), 83.0, 60.0, 53.1, 46.4, 42.7, $20.4\left(-\mathrm{CH}_{3}\right), 14.5\left(-\mathrm{CH}_{3}\right), 14.4\left(-\mathrm{CH}_{3}\right)$; HRMS (ESI) (m/z) Calcd for $\mathrm{C}_{21} \mathrm{H}_{26} \mathrm{~N}_{2} \mathrm{O}_{5} \mathrm{~S} 419.16352$ found $419.16443(\mathrm{M}+\mathrm{H})^{+}$

Ethyl-2,5-dimethyl-5-(4-(3-(thiophen-2-yl)but-2-enoyl)piperazine-1-carbonyl)-4,5dihydrofuran-3-carboxylate (3p)

It was obtained as a yellow oil; yield: 20\% (86 mg); IR (ATR) $v_{\max } 3085,2987,2930,2863$, $1734(\mathrm{C}=\mathrm{O}), 1694(\mathrm{C}=\mathrm{O}), 1620(\mathrm{C}=\mathrm{C}), 1194,1062,761,706($ arom. $\mathrm{CH}) \mathrm{cm}^{-1} ;{ }^{1} \mathrm{H}$ NMR $(400$ $\left.\mathrm{MHz}, \mathrm{CDCl}_{3}\right) \delta(\mathrm{ppm}): 7.20(1 \mathrm{H}, \mathrm{d}, J=0.8 \mathrm{~Hz}$, arom. $\mathrm{CH}), 7.03-7.01(2 \mathrm{H}, \mathrm{m}$, arom. $\mathrm{CH}), 6.37$ $\left(1 \mathrm{H}, \mathrm{s}, \mathrm{H}_{\mathrm{olef}}\right), 4.16\left(2 \mathrm{H}, \mathrm{q}, J=7.2 \mathrm{~Hz},-\mathrm{OC}_{2} \mathrm{CH}_{3}\right), 3.89-3.50(8 \mathrm{H}$, broad $), 3.59(1 \mathrm{H}, \mathrm{d}, J=15.2$ Hz, Ha-4), $2.72(1 \mathrm{H}, \mathrm{d}, J=15.2 \mathrm{~Hz}, \mathrm{Hb}-4), 2.33\left(3 \mathrm{H}, \mathrm{s},-\mathrm{CH}_{3}\right), 2.26\left(3 \mathrm{H}, \mathrm{s},-\mathrm{CH}_{3}\right), 1.58(3 \mathrm{H}, \mathrm{s}$, $\left.-\mathrm{CH}_{3}\right), 1.27\left(3 \mathrm{H}, \mathrm{t}, J=7.2 \mathrm{~Hz},-\mathrm{OCH}_{2} \underline{\mathrm{C}}_{3}\right) ;{ }^{13} \mathrm{C}-\mathrm{NMR}\left(100 \mathrm{MHz}, \mathrm{CDCl}_{3}\right), \delta(\mathrm{ppm}): 170.5$ $(\mathrm{C}=\mathrm{C}, \mathrm{C}-2), 166.6(\mathrm{C}=\mathrm{O}), 165.6(\mathrm{C}=\mathrm{O}), 164.5(\mathrm{C}=\mathrm{O}), 145.1(\mathrm{C}=\mathrm{C}), 140.6,127.8,126.9,125.8$, $116.25(\mathrm{C}=\mathrm{C}), 102.3(\mathrm{C}=\mathrm{C}, \mathrm{C}-3), 88.3,59.7,46.3,43.5,41.2,26.04\left(-\mathrm{CH}_{3}\right), 17.83\left(-\mathrm{CH}_{3}\right), 14.36$ (- $\left.\mathrm{CH}_{3}\right), 14.07\left(-\mathrm{CH}_{3}\right)$; HRMS (ESI) (m/z) Calcd for $\mathrm{C}_{22} \mathrm{H}_{28} \mathrm{~N}_{2} \mathrm{O}_{5} \mathrm{~S} 433.17917$ found 433.18051 $(\mathrm{M}+\mathrm{H})^{+}$

\section{Method of in vitro $\mathrm{AChE}$ inhibition experiments}

Slightly modified Ellman method was carried out to determine in vitro AChE inhibitory activities of test compounds [57].

The assay solution was prepared by adding $1480 \mu \mathrm{L}$ of phosphate buffer $(\mathrm{pH}=8.0,0.1 \mathrm{M}), 50$ $\mu \mathrm{L}$ of DTNB solution (prepared with $\mathrm{pH} 7$ phosphate buffer), $20 \mu \mathrm{L}$ of test compounds at desired concentration in ethanol-deionized water (1:1), $10 \mu \mathrm{L}$ of substrate solution (ATCI, in deionized water) and $25 \mu \mathrm{L}$ of AChE solution (prepared with deionized water and $1 \%$ gelatin). After that assay solution were incubated for $10 \mathrm{~min}$. at $30^{\circ} \mathrm{C}$ and absorbance at $412 \mathrm{~nm}$ were determined.

A control solution containing all compounds except inhibitor was performed same as above and the absorbance at $412 \mathrm{~nm}$ was considered $100 \%$ enzyme activity. 
The percentage activity of AChE for any tested compound at desired concentration was calculated with the formula:,

$$
\% \text { enzyme activity }=\left(\mathrm{A}_{\mathrm{s}} / \mathrm{A}_{0}\right) \times 100
$$

$A_{s}$ : Absorbance of assay solution with inhibitor.

$\mathrm{A}_{0}$ : Absorbance of control solution.

The concentration of each test compound was tested in triplicate and $\mathrm{IC}_{50}$ values were calculated graphically using GraphPad Prism 8.0.3 software. $\mathrm{IC}_{50}$ value is defined as the concentration of sample which performs 50\% inhibition towards AChE.

\section{Methods of in silico molecular docking experiments}

Three dimensional structure of recombinant human AChE complexed with donepezil was obtained from the Protein Data Bank (4EY7) [58]. B-chain, water molecules and detergents were removed. Conformational analysis of inhibitor test compounds were performed with Avogadro software and most stable conformations were optimized with semiempirical PM6 method in Gaussian 09 Software. All ligand-protein docking calculations were performed as flexible ligand in rigid protein using AutoDock Vina software [59]. Best docking mod of ligand in terms of binding energy ( $\mathrm{Kcal} / \mathrm{mol})$ was selected and used.

According to these results, it can be seen that all piperazine-dihydrofuran AChEI compounds presents satisfactory druglike properties, including our lead compound $\mathbf{3 j}$. 


\section{Supplementary Material}

Copies of ${ }^{1} \mathrm{H}$ NMR, ${ }^{13} \mathrm{C}$ NMR and HRMS spectra for all novel compounds can be found as supplementary information.

\section{Acknowledgements}

This study was financially supported by the Scientific and Technical Research Council of Turkey (TUBITAK) (TBAG-116Z455). Sait SARI thanks to TUBITAK for doctoral fellowship.

\section{Conflict of Interest}

The authors declare that they have no conflict of interest.

\section{References}

1. Alzheimer's Association. 2017 Alzheimer's disease facts and figures. Alzheimers Dement. 2017;13:325-373.

2. He Q, Liu J, Lan J, Ding J, Sun Y, Fang Y, Jiang N, Yang Z, Sun L, Jin Y, Xie S. Coumarin-dithiocarbamate hybrids as novel multitarget AChE and MAO-B inhibitors against Alzheimer's disease: design, synthesis and biological evaluation. Bioorg Chem. 2018;81:512-528.

3. Wortmann M. World Alzheimer report 2014: dementia and risk reduction. Alzheimers Dement. 2014;11:837.

4. Hampel H, Mesulam MM, Cuello A, Khachaturian AS, Vergallo A, Farlow MR, Snyder PJ, Giacobini E, Khachaturian ZS. Revisiting the Cholinergic Hypothesis in Alzheimer's Disease: Emerging Evidence from Translational and Clinical Research. J Prev Alzheimers Dis. 2019;6:2-15.

5. Mufson EJ, Ikonomovic MD, Counts SE, Perez SE, Ahmadi MM, Scheff WS, Ginsberg SD. Molecular and cellular pathophysiology of preclinical Alzheimer's disease. Behav Brain Res. 2016;311:54-69. 
6. LaFerla F, Green K, Oddo S. Intracellular amyloid- $\beta$ in Alzheimer's disease. Nat Rev Neurosci. 2007;8:499-509.

7. Dumas JA, Newhouse PA. The cholinergic hypothesis of cognitive aging revisited again: cholinergic functional compensation. Pharmacol Biochem Behav. 2011;99:25461.

8. Akasofu S, Kimura M, Kosasa T, Sawada K, Ogura H. Study of neuroprotection of donepezil, a therapy for Alzheimer's disease. Chem Biol Interact. 2008;175:222-226.

9. Molinuevo JL, Gauthier S. Benefits of combined cholinesterase inhibitor and memantine treatment in moderate-severe Alzheimer's disease. Alzheimers Dement. $2013 ; 9: 326-331$

10. Tougu V. Acetylcholinesterase: Mechanism of catalysis and inhibition. Curr Med Chem. 2001;1:155-170.

11. Rashid U, Ansari FL. Challenges in designing therapeutic agents treating Alzheimer's disease-form serendipity to rationality. Drug Design Discovery in Alzheimer's Disease. Elsevier; 2015.

12. Sugimoto H, Iimura Y, Yamanishi Y, Yamatsu K. Synthesis and structure-activity relationships of acetylcholinesterase inhibitors: 1-benzyl-4-[(5,6-dimethoxy-1oxoindan-2-yl)methyl]piperidine hydrochloride and related compounds. J Med Chem. 1995;38:4821-4829.

13. Weintraub D, Somogyi M, Meng X. Rivastigmine in Alzheimer's disease and Parkinson's disease dementia: an ADAS-cog factor analysis. Am J Alzheimers Dis Other Demen. 2011;26:443-449.

14. Giacobini E. Cholinesterase inhibitors: New roles and therapeutic alternatives. Pharmacol Res. 2004;50:433-440. 
15. Taylor AP, Robinson RP, Fobian YM, Blakemore DC, Jones LH, Fadeyi O. Modern advances in heterocyclic chemistry in drug discovery. Org Biomol Chem. 2016;14:66116637.

16. Szabo M, Herenbrink CK, Christopoulos A, Lane JR, Capuano B. Structure-Activity Relationships of Privileged Structures Lead to the Discovery of Novel Biased Ligands at the Dopamine D2 Receptor. J Med Chem. 2014;57:4924-4939.

17. Chapman AG, Hart GP, Meldrum BS, Turski L, Watkins JC. Anticonvulsant activity of two novel piperazine derivatives with potent kainate antagonist activity. Neurosci. 1985;55:325-330.

18. Patil M, Poyil AN, Joshi SD, Patil SA, Patil SA, Bugarin A. Design, synthesis, and molecular docking study of new piperazine derivative as potential antimicrobial agents. Bioorg Chem. 2019;92:103217.

19. Patel KN, Telvekar VN. Design, synthesis and antitubercular evaluation of novel series of $N$-[4-(piperazin-1-yl)phenyl]cinnamamide derivatives. Eur $\mathrm{J}$ Med Chem. $2014 ; 75: 43-56$.

20. Islam I, Hinshaw RR, Chong KT, Kato A, Borchard RT, Fisher JF. Synthesis and Antiviral Activity of [2-[[4-[3-[(1-Methylethyl)amino]-2-Pyridyl]-1Piperazinyl]Carbonyl]-1H-Indol-5-yl] (BHAP) Acylsphingosine HIV Reverse Transcriptase Inhibitors. Bioorg Chem. 1995;23:4499-511.

21. Tugrak M, Gul HI, Bandow K, Sakagami H, Gulcin I, Ozkay Y, Supuran CT. Synthesis and biological evaluation of some new mono Mannich bases with piperazines as possible anticancer agents and carbonic anhydrase inhibitors. Bioorg Chem. 2019;90: 103095. 
22. Pretorius SI, Breytenbach WJ, de Kock C, Smith PJ, N'Da DD. Synthesis, characterization and antimalarial activity of quinoline-pyrimidine hybrids. Bioorg. Med. Chem. 2013;21:269-277.

23. Meena P, Nemaysh V, Khatri M, Manral A, Luthra PM, Tiwari M. Synthesis, biological evaluation and molecular docking study of novel piperidine and piperazine derivatives as multi-targeted agents to treat Alzheimer's disease. Bioorg. Med. Chem. 2015;23: $1135-1148$.

24. Piemontese L, Tomás D, Hiremathad A, Capriati Vi, Candeias E, Cardoso SM, Chaves S, Santos MA. Donepezil structurebased hybrids as potential multifunctional antiAlzheimer's drug candidates. J Enzyme Inhib Med Chem. 2018;33:1212-1224.

25. Demirayak Ş, Şahin Z, Ertaş M, Bülbül EF, Bender C, Biltekin SN, Berk B, Sağlık BN, Levent S, Yurttaş L. Novel thiazole-piperazine derivatives as potential cholinesterase inhibitors. J Heterocyclic Chem. 2019;56:3370-3386.

26. Chen SP, Chen BW, Dai CF, Sung PJ, Wu YC, Sheu JH. Sarcophytonins F and G, New Dihydrofuranocembranoids from a Dongsha Atoll Soft Coral Sarcophyton sp. Bull Chem Soc Jpn. 2012;85:920-922.

27. Lallemand JY, Six Y, Ricard LA. Concise Synthesis of an Advanced Clerodin Intermediate through a Vaultier Tandem Reaction. Eur J Org Chem. 2002;3:503-513.

28. Appendino G, Cravotto G, Palmisano G, Annunziata R. Synthesis of fercoprolone, a degraded prenylated coumarin. Tetrahedron. 1998;54:10819-10826.

29. Kornsakulkarn J, Saepua S, Srichomthong K, Supothina S, Thongpanchang C. New mycotoxins from the scale insect fungus Aschersonia coffeae Henn. BCC 28712. Tetrahedron. 2012;68:8480-8486.

30. Melikyan GG. Manganese(III) Mediated Reactions of Unsaturated Systems. Synthesis $1993 ; 9: 833-850$. 
31. Mondal M, Bora U. Recent advances in manganese(iii) acetate mediated organic synthesis. RSC Adv. 2013;3:18716-18754.

32. Snider BB. Manganese(III)-Based Oxidative Free-Radical Cyclizations. Chem Rev. 1996;96:339-363.

33. Castro S, Fernandez JJ, Fananas FJ, Vicente R, Rodriguez F. Manganese-Mediated CH Alkylation of Unbiased Arenes Using Alkylboronic Acids. Chem Eur J. 2016;22: 9068-9071.

34. Lofstrand VA, Matsuura BS, Furst L, Narayanam JMR, Stephenson JRC. Formation and trapping of azafulvene intermediates derived from manganese-mediated oxidative malonate coupling. Tetrahedron. 2016;72:3775-3780.

35. Aslan H, Öktemer A, Dal H, Hökelek T. Synthesis of ferrocene substituted dihydrofuran derivatives via manganese(III) acetate mediated radical addition-cyclization reactions. Tetrahedron. 2017;73:7223-7232.

36. Hyunh TT, Nguyen VH, Nishino H. One-pot synthesis of 2-oxa-7-azaspiro[4.4]nonane8,9-diones using Mn(III)-based oxidation of 4-acylpyrrolidine-2,3-diones. Tetrahedron Lett. 2017;58:3619-3622.

37. Zhang PZ, Zhang L, Li JA, Shoberu A, Zou JP, Zhang W. Phosphinoyl Radical Initiated Vicinal Cyanophosphinoylation of Alkenes. Org Lett. 2017;19:5537-5540.

38. Kobayashi K, Nagase K, Morikawa O, Konishi H. Convenient Synthesis of Furopyranopyrandione Derivatives by the CAN-mediated Furan Ring Formation. Heterocycles. 2003;60:939-946.

39. Chuang $\mathrm{CP}, \mathrm{Wu}$ YL. Oxidative free radical reactions of enamino esters. Tetrahedron. 2004;60:1841-1847. 
40. Nair V, Mohanan K, Suja TD, Suresh E. Stereoselective synthesis of 3,4-transdisubstituted pyrrolidines and cyclopentanes via intramolecular radical cyclizations mediated by CAN. Tetrahedron Lett. 2006;47:2803-2806.

41. Hocaoglu B, Yilmaz M. Regioselective radical addition of 3- oxopropanenitriles with terminal dienes promoted by cerium(IV) ammonium nitrate and manganese(III) acetate. Synth Commun. 2019;49:1938-1946.

42. Ustalar A, Yilmaz M. Microwave assisted synthesis of 2,3-dihydro-4Hbenzo[4,5]thiazolo[3,2- $a]$ furo[2,3- $d]$ pyrimidin-4-ones and 6,7-dihydro-5H-furo[2,3d] thiazolo[3,2-a]pyrimidin-5-ones using $\mathrm{Mn}(\mathrm{OAc})_{3}$. Tetrahedron Lett. 2017;58:516519.

43. Yilmaz M, Bicer E, Ustalar A, Pekel AT. Synthesis of furan-substituted dihydrofuran compounds by radicalcyclization reactions mediated by manganese(III) acetate. Arkivoc. 2014;(v):225-236.

44. Özgür M, Yılmaz M, Nishino H, Avar EÇ, Dal H, Pekel AT, Hökelek T. Efficient syntheses and antimicrobial activities of new thiophene containing pyranone and quinolinone derivatives using manganese(III) acetate: the effect of thiophene on ring closure-opening reactions. New J Chem. 2019;43:5737-5751.

45. Yılmaz EVB, Y1lmaz M, Öktemer A. Radical cyclizations of conjugated esters and amides with 3-oxopropanenitriles mediated by manganese(III) acetate. Arkivoc. 2011;(ii):363-376.

46. Yilmaz M, Ustalar A, Uçan B, Pekel AT. Regio- and diastereoselective synthesis of trans-dihydrofuran-3-carboxamides by radical addition of 1,3-dicarbonyl compounds to acrylamides using manganese(III) acetate and determination of exact configuration by X-ray crystallography. Arkivoc. 2016;(vi):79-91. 
47. Pan W, Hu K, Bai P, Yu L, Ma Q, Li T, Zhang X, Chen C, Peng K, Liu W, Sang Z. Design, synthesis and evaluation of novel ferulic acid-memoquin hybrids as potential multifunctional agents for the treatment of Alzheimer's disease. Bioorg Med Chem Lett. 2016;26(10):2539-2543.

48. Shaik JB, Yeggoni DP, Kandrakonda YR, Penumala M, Zinka RB, Kotapati KV, Darla MM, Ampasala DR, Subramanyam R, Amooru DG. Synthesis and biological evaluation of flavone-8-acrylamide derivatives as potential multi-target-directed anti Alzheimer agents and investigation of binding mechanism with acetylcholinesterase. Bioorg Chem. 2019;88:102960.

49. Estrada M, Herrera-Arozamena C, Perez C, Vina D, Romero A, Morales-Garcia JA, Castillo AP, Rodriguez-Franco MI. New cinnamic $-N$-benzylpiperidine and cinnamic - N,N-dibenzyl( $N$-methyl)amine hybrids as Alzheimer-directed multitarget drugs with antioxidant, cholinergic, neuroprotective and neurogenic properties. Eur J Med Chem. 2016;121:376-386.

50. Singh YP, Tej GNVC, Pandey A, Priya K, Pandey P, Shankar G, Nayak PK, Rai G, Chittiboyina AG, Doerksen RJ, Vishwakarma S, Modi G. Design, synthesis and biological evaluation of novel naturally-inspired multifunctional molecules for the management of Alzheimer's disease. Eur J Med Chem. 2020;198:112257.

51. Sari S, Yilmaz M. Synthesis, characterization, acetylcholinesterase inhibition, and molecular docking studies of new piperazine substituted dihydrofuran compounds. Med Chem Res. 2020;29:1804-1818.

52. Snider BB. Manganese(III)-Based Oxidative Free-Radical Cyclizations. Chem Rev. 1996;96:339-363.

53. Fu J, Bao F, Gu M, Liu J, Zhang Z, Ding J, Xie S, Ding J. Design, synthesis and evaluation of quinolinone derivatives containing dithiocarbamate moiety as 
multifunctional AChE inhibitors for the treatment of Alzheimer's disease. J Enzyme Inhib. 2020;35:118-128.

54. Lipinski CA, Lombardo F, Dominy BW, Feeney PJ. Experimental and computational approaches to estimate solubility and permeability in drug discovery and development settings. Adv Drug Deliv Rev. 1997;23:3-25.

55. Lipinski CA, Lombardo F, Dominy BW, Feeney PJ. Experimental and computational approaches to estimate solubility in drug discovery and development settings. Adv Drug Deliv Rev. 2001;46:3-26.

56. Sarı S, Ünalan S, Y1lmaz M. Synthesis and characterization of unsaturated diacyl and alkyl-acyl piperazine derivatives. Turk J Chem. 2019;43:1656-1671.

57. Ellman GL, Courtney KD, Andres Jr V, Featherstone RM. A new and rapid colorimetric determination of acetylcholinesterase activity. Biochem Pharmacol. 1961;7:90-95.

58. Cheung J, Rudolph MJ, Burshteyn F, Cassidy MS, Gary EN, Love J, Franklin MC, Height JJ. Structures of human acetylcholinesterase in complex with pharmacologically important ligands. J Med Chem. 2012;55:10282-10286.

59. Trott O, Olson AJ. AutoDock Vina: improving the speed and accuracy of docking with a new scoring function, efficient optimization and multithreading. J Comput Chem. 2010;31:455-461. 
Figures

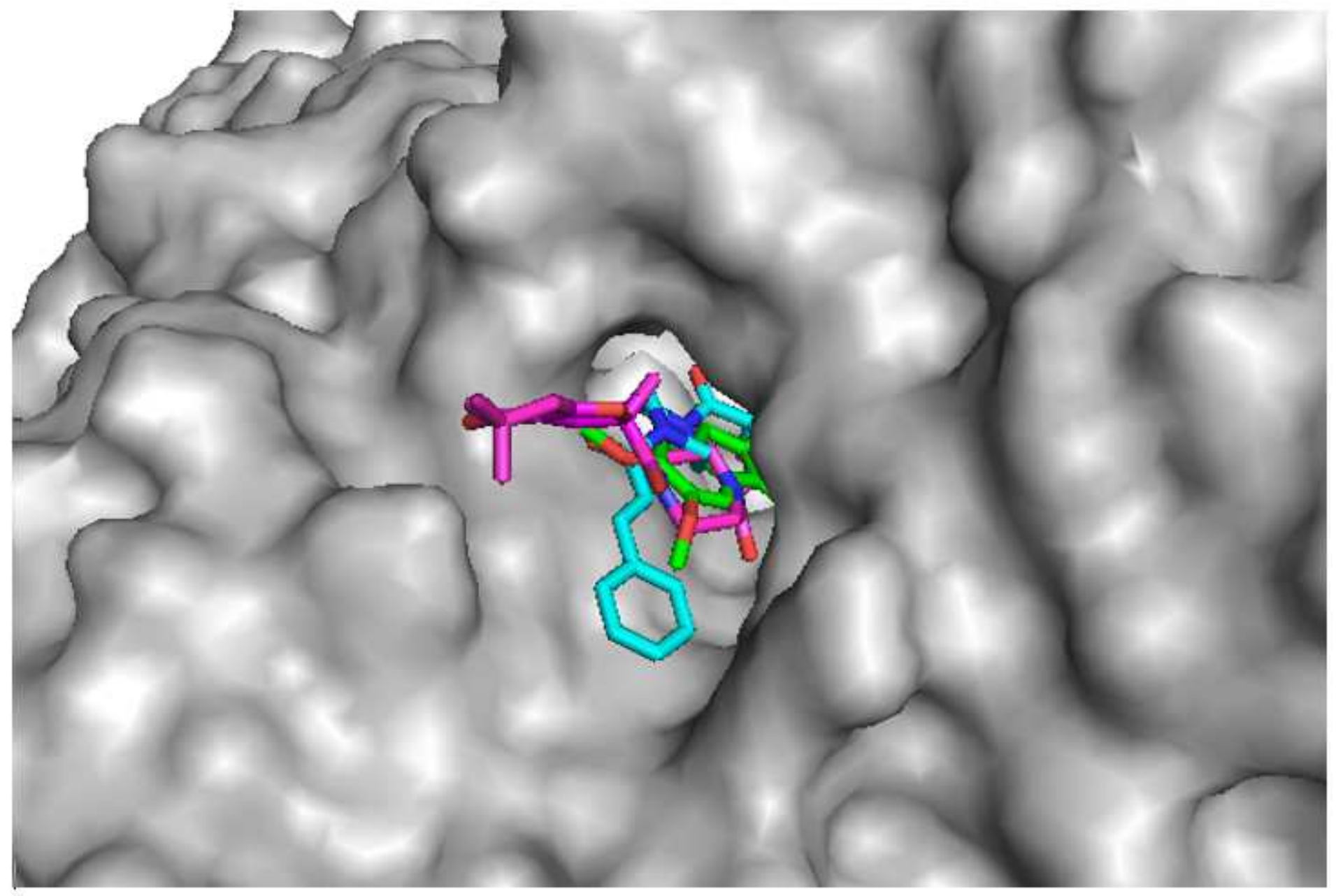

Figure 1

AChE active site cavity with donepezil (green), 3a (magenta) and 3j (cyan) inside. 


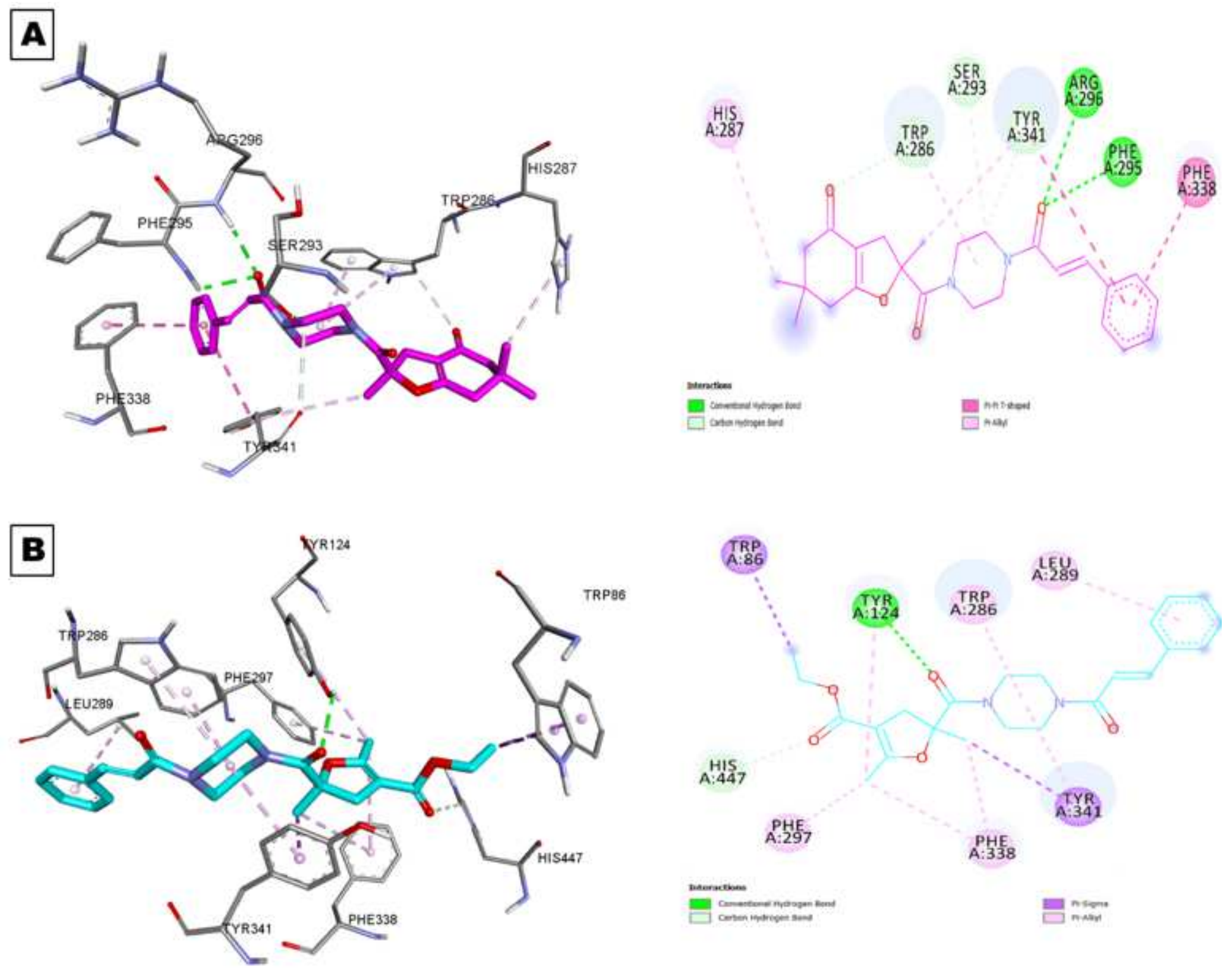

Figure 2

Ligand-protein interactions of $3 a(A)$ and $3 j(B)$.

\section{Supplementary Files}

This is a list of supplementary files associated with this preprint. Click to download.

- Graphicalabstract.tif

- SupplementaryMaterial.pdf

- Scheme1.png

- Scheme2.png 